\title{
DISCRETE CHARACTERIZATION OF COHESION IN GAS-SOLID FLOWS
}

\author{
by \\ Kunal Jain \\ B.E. in Ch. E., Panjab University, India, 2000 \\ Submitted to the Graduate Faculty of \\ the School of Engineering in partial fulfillment \\ of the requirement for the degree of \\ Master of Science in Chemical Engineering
}

University of Pittsburgh 


\section{UNIVERSITY OF PITTSBURGH}

School of Engineering

This thesis was presented

by

\section{Kunal Jain}

It was defended on

August 26, 2002

and approved by

Thesis Advisor: Joseph. J. McCarthy, Assistant Professor, Chemical and Petroleum Engineering Department 


\title{
ABSTRACT \\ DISCRETE CHARACTERIZATION OF COHESION IN GAS-SOLID FLOWS
}

\author{
Kunal Jain , M.S. \\ University of Pittsburgh
}

Fluidization and the transport of solid particles either by gravity or by pneumatic means are used in a variety of industrial operations, including fluid catalytic cracking, fluid hydroforming and solid fuel processes such as coal gasification and liquefaction ${ }^{[1] *}$. Despite the fact that a sizeable portion of gas-solid flows are cohesive in nature, the mechanics of cohesive flowing gas-particle systems is still poorly understood, and manipulation/control of the flow variables is still largely done on a trial-and-error basis.

Cohesive forces between grains can arise from a variety of sources - such as liquid bridge (capillary) forces, van der Waals forces, or electrostatic forces - and may play a significant role in the processing of fine and/or moist powders. While recent advances have been made in our understanding of liquid-induced cohesion in quasi-static systems at the macroscopic level ${ }^{[2,3]}$, in general, it is still not possible to directly connect this macroscopic understanding of cohesion with a microscopic picture of the particle properties and interaction

\footnotetext{
${ }^{*}$ Bracketed references placed superior to the line of text refer to the bibliography.
} 
level ${ }^{[2,3]}$, in general, it is still not possible to directly connect this macroscopic understanding of cohesion with a microscopic picture of the particle properties and interaction forces. Moreover, conventional theories on gas-solid flows, make no attempt to distinguish between these modes of cohesion, despite clear qualitative differences (lubrication forces in wet systems or electrostatic repulsion are two good examples).

In this work, we extend the previous work on discrete characterization tools of wet granular flows ${ }^{[4]}$, using computations of gas-solid flows, in order to examine the transition from non-cohesive (dry) to cohesive (wet) behavior in gas-solid systems. Gas velocity and bridging liquid surface tension are varied to explore a range of the possible fluidization parameter space and a characterization criterion based on the the physical picture of liquid-induced particle-level cohesion is developed for gas-solid flows. Cohesion between wet particles has been modeled using the concept of liquid bridges. The characterization tool developed, namely the Granular Capillary Number, is tested by measuring both the minimum fluidization velocity as well as the mixing rate in fluidized systems. The systems explored here are traditionally thought to be cohesive but a marked difference is observed as the Granular Capillary Number changes.

\section{DESCRIPTORS}

Gas-solid Flows

Particle Dynamics

Cohesion
Fluidization

CFD

Liquid Bridges 


\section{ACKNOWLEDGMENTS}

I would like to thank my advisor, Prof. J. J. McCarthy, for his support, patience and invaluable guidance throughout my graduate studies. I'm also thankful to my committee members Prof. Robert Enick and Prof. Robert Parker for their feedback and suggestions. Special thanks are due to John Harrold and Watson Vargas for always helping me whenever I ran into problems. I would also like to acknowledge all the past and current inhabitants

of my lab for their help and friendship: Adetola Abatan, Aditya Bedekar, Lei Hong, Philip Lenart, Hongming Li and Abhishek Soni.

Finally, I would like to thank my family for their support and understanding and to all the people that have supported and helped me over the years. 


\section{TABLE OF CONTENTS}

$\underline{\text { Page }}$

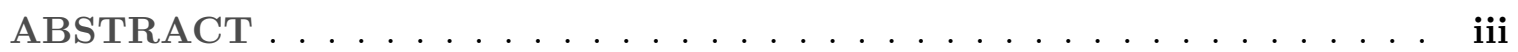

ACKNOWLEDGMENTS . . . . . . . . . . . . . . . v

LIST OF TABLES . . . . . . . . . . . . . . . . . . . viii

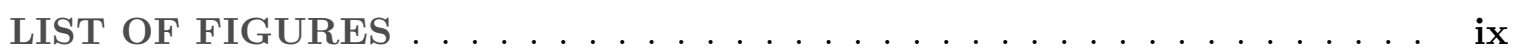

$1.0 \quad$ INTRODUCTION $\ldots \ldots \ldots \ldots \ldots \ldots \ldots \ldots \ldots \ldots$

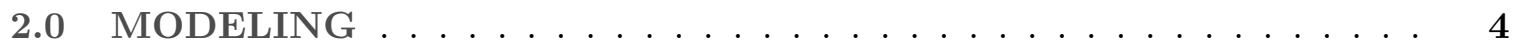

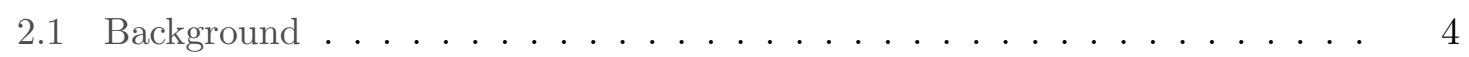

2.2 Particle Dynamics . . . . . . . . . . . . . . . . 5

$2.2 .1 \quad$ Capillary Forces . . . . . . . . . . . . . . . . 10

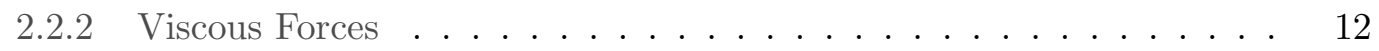

2.2 .3 Drag Force $\ldots \ldots \ldots \ldots \ldots \ldots \ldots \ldots \ldots \ldots$

2.3 Fluid Dynamics . . . . . . . . . . . . . . . . . 15

3.0 CODE DEVELOPMENT $\ldots \ldots \ldots \ldots \ldots \ldots \ldots \ldots$

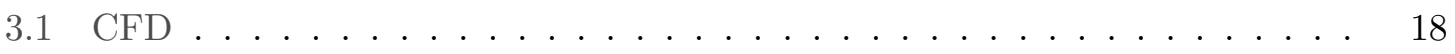

3.1 .1 Discretization . . . . . . . . . . . . . . . . . . . . 19

$3.1 .2 \quad$ SIMPLE . . . . . . . . . . . . . . . . 23

3.2 Particle Dynamics . . . . . . . . . . . . . . . . 26 
4.0 CHARACTERIZATION TOOLS . . . . . . . . . . . . . 31

4.1 Granular Capillary Number . . . . . . . . . . . . . . . . . . . . . 31

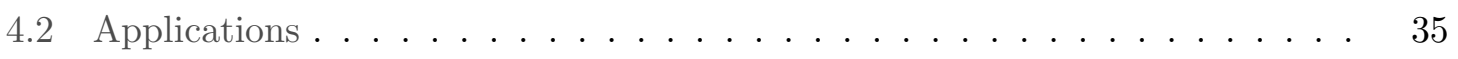

4.2 .1 Mixing . . . . . . . . . . . . . . . . . 37

4.2 .2 Fluidization . . . . . . . . . . . . . . . 41

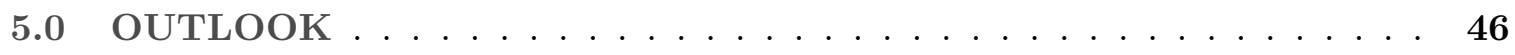

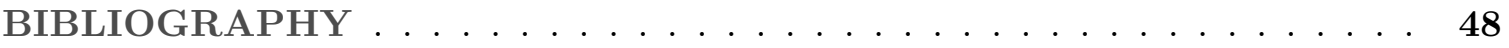




\section{LIST OF TABLES}

Table No.

$\underline{\text { Page }}$

4.1 Parameters used in the simulation . . . . . . . . . . . . . 35 


\section{LIST OF FIGURES}

Figure No. $\quad$ Page

1.1 Geldart's classification . . . . . . . . . . . . . . . . . . . 3

2.1 Contact point between two particles. . . . . . . . . . . . . . 6

2.2 Models of contact force . . . . . . . . . . . . . . . . . 7

2.3 Types of liquid bridges . . . . . . . . . . . . . . . . . . . . 11

2.4 Schematic of a symmetric liquid bridge . . . . . . . . . . . . . 13

2.5 Boundary Conditions . . . . . . . . . . . . . . . . . . . 17

3.1 Staggered Grid . . . . . . . . . . . . . . . . . 20

3.2 No Binary Search Grid. . . . . . . . . . . . . . . . . . . . . 27

3.3 Comparison of contact-detection algorithms. . . . . . . . . . . . . . 30

4.1 Characterization via the Granular Bond Number . . . . . . . . . . . . . . 32

4.2 Characterization via the Collision Number . . . . . . . . . . . . . . . 34

4.3 Mixing progress for Cohesive/Non-Cohesive materials $\ldots \ldots \ldots$

4.4 Mixing Rates at different gas velocities . . . . . . . . . . . . . . . 38

4.5 Mixing Rates at different surface tensions . . . . . . . . . . . . . . . . 39

4.6 Mixing Rate versus Granular Capillary Number . . . . . . . . . . . . . . . . 40

4.7 Pressure Drop versus Time for different gas velocities . . . . . . . . . . . . . 42

4.8 Standard Deviation of the Pressure Drop versus Gas-Velocity . . . . . . . . 43

4.9 \%change in minimum fluidization velocity versus Granular Capillary Number 44 


\subsection{INTRODUCTION}

It has been estimated that approximately $\$ 61$ billion in the U.S. chemical industry is

linked to particle technology ${ }^{[5]}$. In particular, gas-solid transport is crucial in a variety of industrially important applications, ranging from fluidized-bed reactors and dryers to pneumatic conveyors and classifiers to spray dryers and coaters. In many instances the cohesive nature of a powder sample is a prime factor contributing to difficulties in proper plant operation where powder flow-ability may cause, for example, channeling and defluidization in combustion/feeder systems. The Rand Corporation conducted a six-year study of 40 solids processing plants in the U.S. and Canada ${ }^{[6]}$. Their study revealed that almost $80 \%$ of these plants experienced solids handling problems. Hence, the problems arising due to the cohesive nature of powders concern a diverse range of industries. Despite recent advances $[2,3]$, an understanding of the flow and characterization of cohesive gas-solid flows remains poor.

The most widely used classification system for particles in gas-solid flows was introduced by Geldart ${ }^{[7]}$ based on the density difference between the particles and the gas $\left(\rho_{p}-\rho_{g}\right)$ and the average particle diameter $d_{p}$ (Figure 1.1). Powders which when fluidized by air at ambient conditions give a region of non-bubbling fluidization beginning at the minimum fluidization velocity followed by bubbling fluidization as fluidizing velocity increases are classified as Group A. Powders which under these conditions give only bubbling fluidization are classified as Group B. Geldart identified two further groups; Group C powders - very fine, cohesive powders which are incapable of fluidization in the strict sense, and Group D powders - large particles distinguished by their ability to produce deep spouting beds. This semi-theoretical classification is ostensibly based on van der Waals cohesion; however a first principles basis for the group boundaries and/or how they change as a function of particle interaction forces is elusive. In particular, the group $\mathrm{A} / \mathrm{C}$ demarcation is by no 
means absolute and in addition to the particle size and density, also shows a dependence on a multiplicity of other factors such as the width of the particle size distribution, the particle shape and the surface texture and composition ${ }^{[8]}$. Recently, computational studies of gasfluidized beds $[2,3,9,10]$ based on the combined approach of discrete element modeling and computational fluid dynamics have been used to investigate the impact of liquid-induced cohesion in these systems. But, the tools available to characterize the flow are not generic. An improved understanding of the role of inter-particle forces in gas-solid flows is essential for improvements in the industrial processes with regards to quality control, equipment design, improved material handling efficiency, etc.

In this work, we discuss several discrete characterization tools for wet (cohesive) granular material with simple, physically relevant interpretations and also examine the utility of these tools by exploring a range of cohesive strengths (from cohesionless to cohesive) in several prototypical applications of solid and gas-solid flows. The document is organized as follows. Chapter 2 gives a brief discussion of background relevant to modeling of gassolid flows. We also describe the Particle Dynamics method and the various inter-particle forces necessary to model liquid-induced cohesion. We finish the section with a description of Fluid Dynamics modeling. Chapter 3 describes briefly the numerical schemes used in the code development. In Chapter 4, characterization criterion based on the the physical picture of liquid-induced particle-level cohesion is reviewed for static and sheared beds and is developed for gas-solid flows. We test the characterization tools by measuring both the minimum fluidization velocity as well as the mixing rate in fluidized systems. Finally, in Chapter 5, limitations and potential extensions of the current work are examined. 


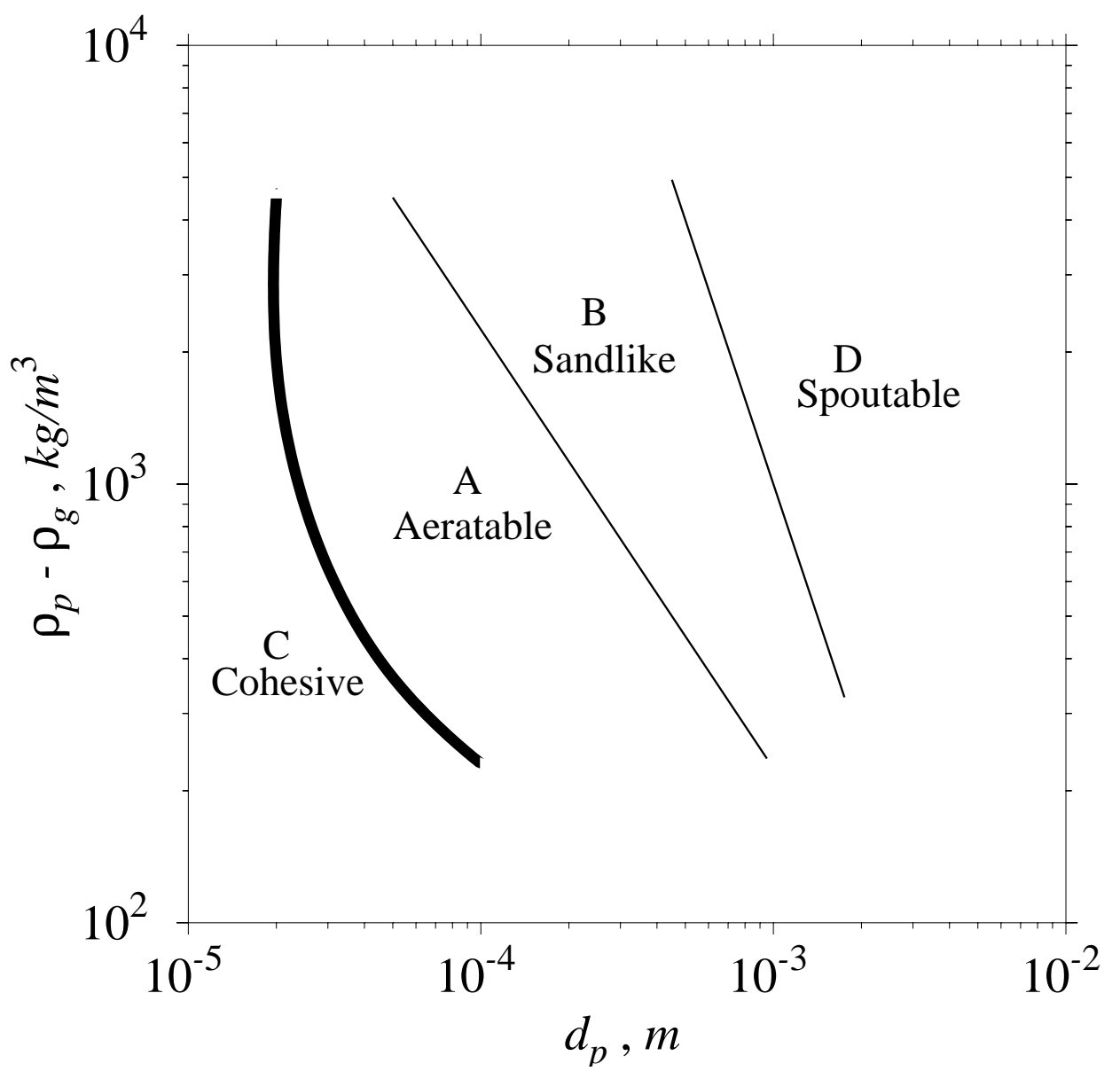

Figure 1.1 Geldart's classification of fluidized particles 


\subsection{MODELING}

\section{$2.1 \quad$ Background}

Two-fluid models and discrete particle simulation are the commonly used methods of simulating gas-solid flows. Two-fluid models ${ }^{[11,12,13]}$ use the Eulerian/continuum approach for the gas as well as the solid phase. The equations developed for the fluid phase (Navier-Stokes equation) are applied to the solid phase with little modification. But the constitutive equations for solid-phase stress description rely strongly on empirical relations

and lack generality ${ }^{[14]}$. In addition to the difficulty of constitutive equations, this approach is unable to model the discrete flow characteristics of individual particles making it impossible to study bed dynamics at the particle level. Concepts from the kinetic theory of gases $[15,16,17]$ have also been extended to describe the stresses. However the same equations run into problems for multiple-particle collisions and the fact that contacts may last a finite interval of time.

Discrete particle simulation is a combined approach where the solid phase is given a Lagrangian treatment and the fluid phase an Eulerian treatment. The solid phase can be modeled using either the soft sphere model or a hard sphere model. The hard sphere model works well in the grain-inertial regime which is dominated by binary collision. This computation is performed by creating an initial condition including the initial positions and velocities for each particle. The time of the first collision for each particle is calculated and the collisions are placed in a sequential order. The simulation then simply steps through the collision list updating it whenever necessary. The soft sphere approach works well in the quasi-static regime where lasting particle contacts are evident. Cundall and Strack's [18] Discrete Element Method (DEM) - also known as Particle Dynamics (PD) - is the commonly used soft sphere model and has been used by several researchers $[9,10,14,19]$ to 
model the solid phase in gas-solid flows. The model is discussed in detail in the following section.

\section{$2.2 \quad$ Particle Dynamics}

Particle Dynamics, a discrete method of simulation, has emerged as one of the most important tools in probing granular flows ${ }^{[10]}$. The method is extremely general in that Newton's second law of motion is used to determine the trajectories of individual particles and the time evolution of these trajectories then determines the global flow of the granular material. The equations that describe the particle motion, therefore are:

Linear Motion:

$$
m_{p} \frac{d v_{p}}{d t}=-m_{p} g+F_{n}+F_{t}
$$

Angular Motion:

$$
I_{p} \frac{d \omega_{p}}{d t}=F_{t} \times R
$$

where $F_{n}$ and $F_{t}$ are the normal and tangential forces acting on the particle and are functions of contact, drag and capillary forces. The particle-to-particle interaction is established by allowing the particles to overlap at the contact point (Figure 2.1). This overlap serves as a parameter in contact mechanics models used to determine the resultant contact force. Cundall and Strack ${ }^{[18]}$ first formulated their force model accounting for the contact mechanics through the use of a spring, a dash-pot and a slider configuration, as shown in Figure 2.2.

The key feature of PD is that many simultaneous two-body interactions may be used to model a many-body system; however care must be taken while choosing the time step. Generally the time-step chosen is smaller than $\frac{R}{\lambda}$, where $R$ is the particle radius and $\lambda$ represents the relevant disturbance wave speed (dilational, distortional or Rayleigh waves 

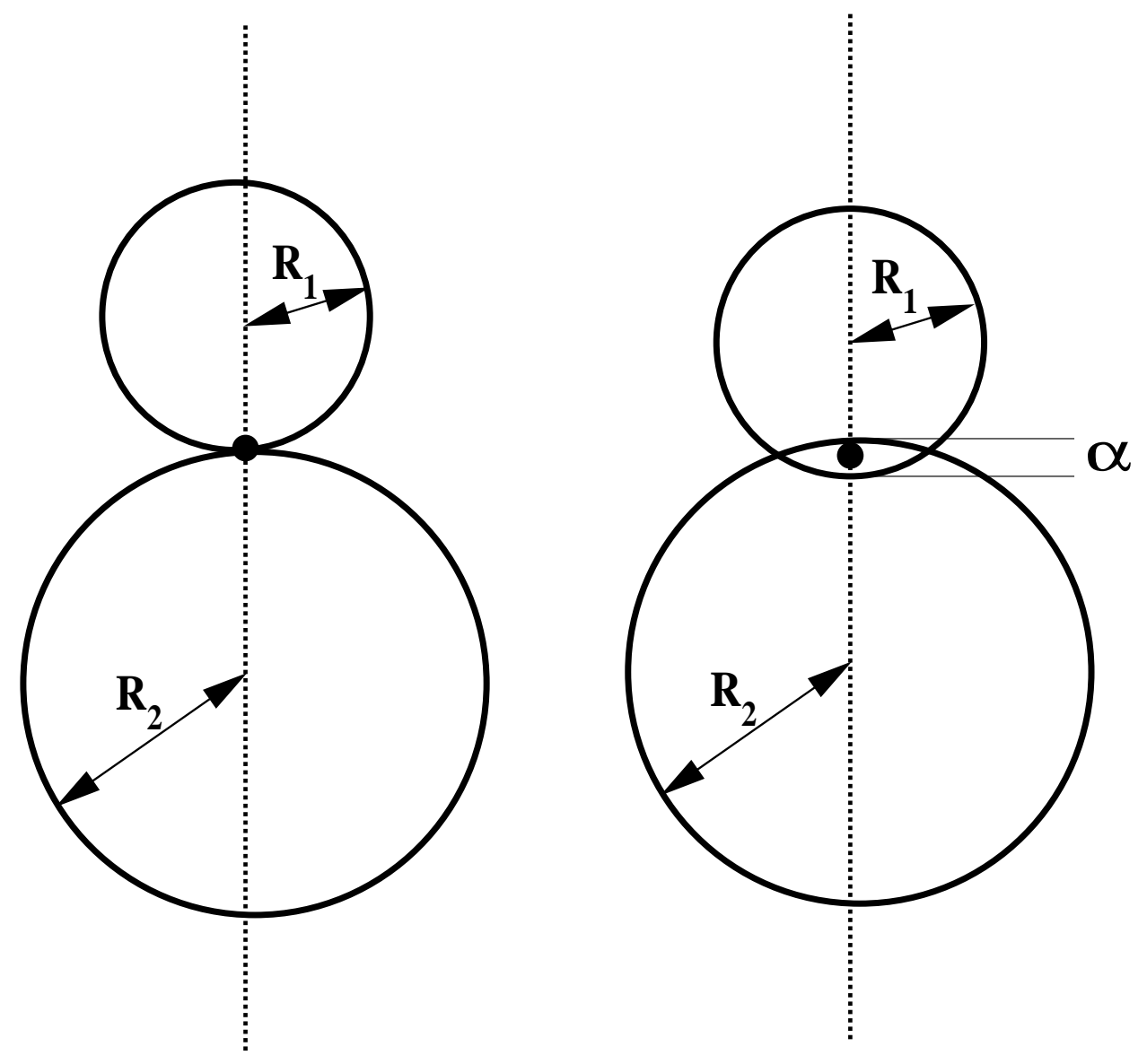

Figure 2.1 Contact point between two particles, showing definition of the overlap $(\alpha)$ 


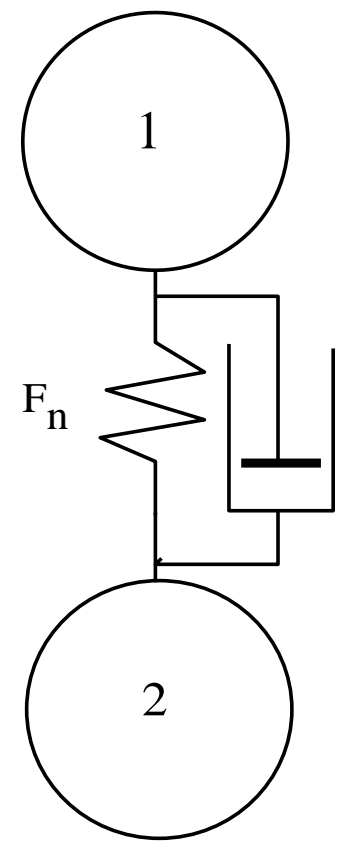

Compressive force

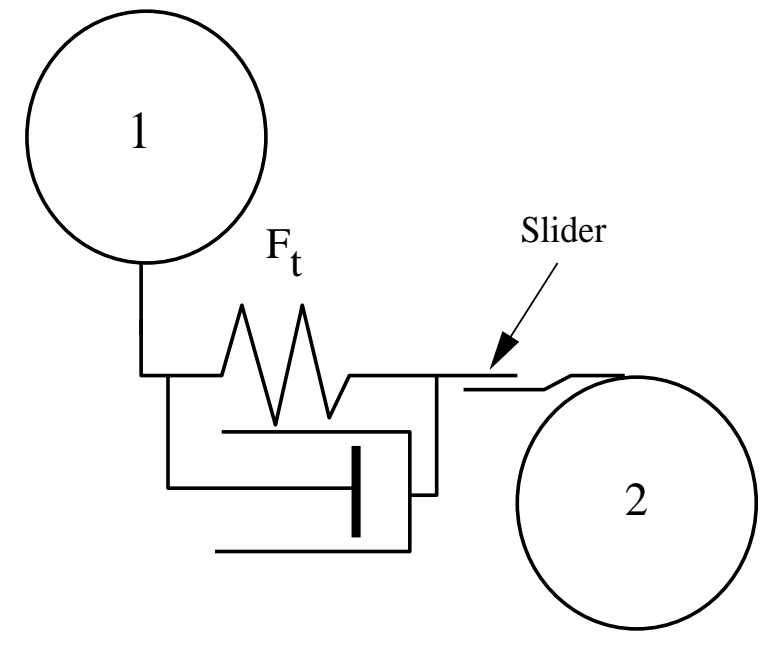

Shearing force

Figure 2.2 Models of contact force. Schematic illustration of the forces - e.g., compressive and shearing - acting on particle 1 from contacting particle 2 . 
${ }^{[20]}$. Under these conditions, the method becomes explicit, and therefore at any time increment the resultant forces on any particle are determined exclusively by its interaction with the closest neighbors in contact. With the accelerations known, the velocities and displacements may be obtained by numerical integration using a finite differences scheme.

The inter-particle forces for cohesionless interactions are typically determined from contact mechanics considerations. In their simplest form, these relations include normal Hertzian repulsion and some approximation of tangential friction. For a thorough description of the possible contact mechanics implementations the reader is referred to Schäfer et al. ${ }^{[21]}$. In the present work, normal interactions are modeled as elastic with a viscous dissipation ${ }^{[18]}$. The normal force, $F_{n}$, is given by:

$$
F_{n}=k_{n} \alpha^{3 / 2}-\eta_{n} v_{n_{12}} \alpha
$$

where $\alpha$ is the deformation of the particles (computationally an "overlap" given by $\alpha=$ $\left(R_{1}+R_{2}\right)-D_{12}$ where $R_{i}$ is the particle radius and $D$ is the distance between particle centers), $k_{n}$ is the normal force constant from the Hertz theory ${ }^{[22]}, \eta_{n}$ is the damping coefficient in the normal direction and $v_{n_{12}}$ is the relative normal velocity of approach of the two particles. The constant $k_{n}$ is related to the particle radii, $R_{i}$, and elastic properties (Young's modulus, $E_{i}$, and Poisson ratio, $\nu_{i}$ ) by:

$$
k_{n}=\frac{4}{3} E^{*} \sqrt{R^{*}}
$$

where $R^{*}$ and $E^{*}$ are:

$$
\begin{gathered}
\frac{1}{E^{*}}=\frac{1-\nu_{1}^{2}}{E_{1}}+\frac{1-\nu_{2}^{2}}{E_{2}} \\
\frac{1}{R^{*}}=\frac{1}{R_{1}}+\frac{1}{R_{2}} .
\end{gathered}
$$


respectively.

The damping coefficient $\eta_{n}$, as proposed by Cundall and Strack ${ }^{[18]}$ is given as:

$$
\eta_{n}=2 \sqrt{m k_{n}}
$$

where $m$ is the mass of the particle. The equation has been derived from the condition of the critical damping of the single degree-of-freedom system consisting of a mass, spring and dash-pot.

The tangential or frictional force is derived from Mindlin ${ }^{[23]}$. At any time-step, the new tangential force acting at a particle-particle contact, $F_{t}$, is given by the sum of the old tangential force, $F_{t_{o}}$, and the incremental change in the tangential force - due to motion during the present time-step. This yields:

$$
F_{t}=F_{t_{o}}-k_{t} \Delta s
$$

where $\Delta s$ is the displacement during the present time-step and is easily calculated from the component of velocity tangent to the contact surface, $v_{t}$ (i.e., $\Delta s=v_{t} d t$ where $d t$ is the time-step). The frictional stiffness, $k_{t}$, is given by:

$$
k_{t}=\frac{2 \sqrt{2 R^{*}} G^{*}}{2-\nu} \sqrt{\alpha}
$$

where $G^{*}$ is a function of Shear modulus $\left(G_{i}\right)$ and Poisson Ratio $\left(\nu_{i}\right)$ given by:

$$
\begin{gathered}
\frac{1}{G^{*}}=\frac{1-\nu_{1}^{2}}{G_{1}}+\frac{1-\nu_{2}^{2}}{G_{2}} \\
G_{i}=\frac{E_{i}}{2\left(1+\nu_{i}\right)} .
\end{gathered}
$$

The overall tangential force is limited by Amonton's Law limit $\left(F_{t} \leq \mu_{f} F_{n}\right.$ where $\mu_{f}$ is the coefficient of sliding friction). For $F_{t} \geq \mu_{f} F_{n}$ the tangential force is given by:

$$
F_{t}=\mu_{f} F_{n}
$$




\subsubsection{Capillary Forces}

Moisture is a common cause of cohesion in gas-solid flows and the forces arising due to the same have been modeled using the concept of liquid bridges. The amount of moisture/liquid determines the degree of saturation which may be characterized as pendular, funicular, capillary and droplet (see Figure 2.3). The pendular regime assumes the saturation is low enough that discrete binary bridges are present between solid surfaces. Several models based

on the solution of the Young-Laplace equation are available in the literature $[9,10,24]$. The capillary force, $F_{c}$, due to both the surface tension of the bridge fluid as well as the pressure difference arising from neck curvature may be expressed as:

$$
F_{c}=2 \pi r_{2} \gamma \sin \beta \sin (\beta+\theta)+\pi R^{2} \Delta P \sin ^{2} \beta
$$

where $r_{2}$ is the bridge neck radius, $\beta$ is the half filling angle, $\theta$ is the contact angle, $\gamma$ is the fluid's surface tension and $\Delta P$ is the pressure difference across the air-liquid interface (see Figure 2.4). This pressure reduction across the capillary is given by the Laplace equation:

$$
\Delta P=\gamma\left[\frac{1}{r_{1}}-\frac{1}{r_{2}}\right]
$$

where $r_{1}$ is the bridge meridional radius of curvature. Mikami et al. ${ }^{[10]}$ provide an empirical fit to the numerical solution of the Laplace-Young equations expressed as:

$$
\begin{gathered}
\hat{F}=\exp (A \hat{h}+B)+C \\
A=-1.1 \hat{V}^{-0.53} \\
B=(-0.34 \ln \hat{V}-0.96) \theta^{2}-0.019 \ln \hat{V}+0.48 \\
C=0.0042 \ln \hat{V}+0.0078
\end{gathered}
$$




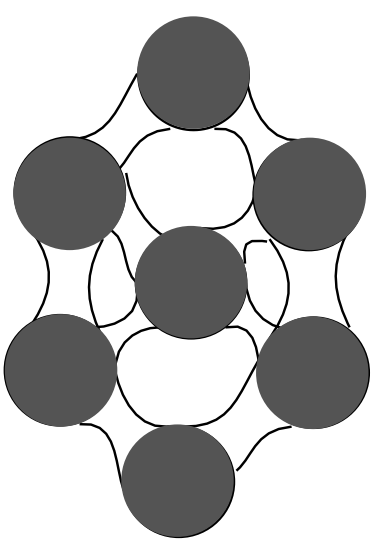

(a)

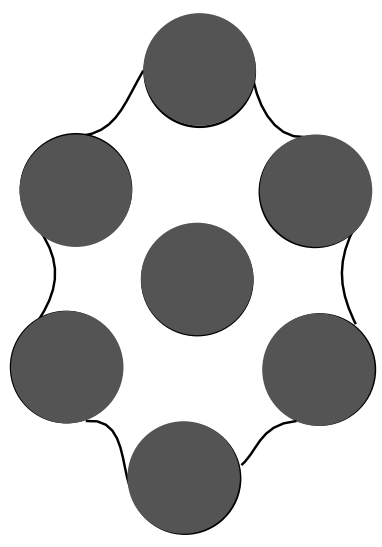

(c)

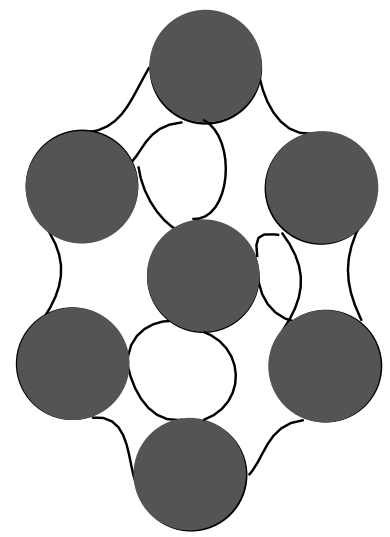

(b)

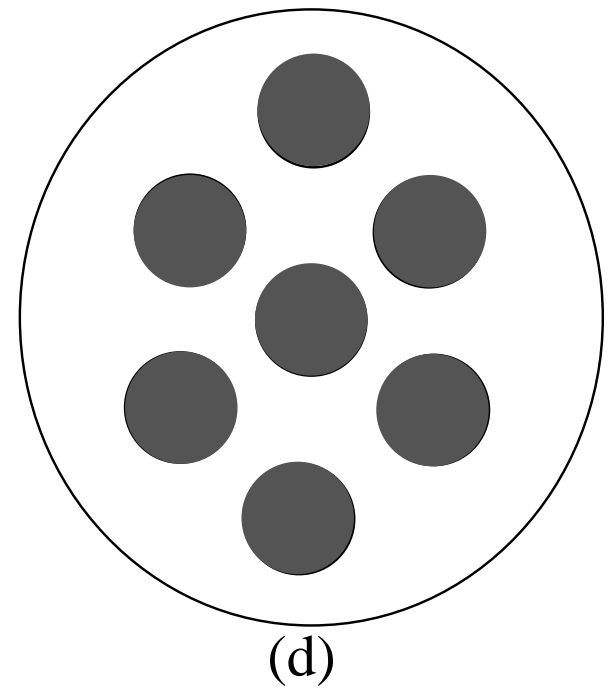

Figure 2.3 Degrees of liquid saturation: (a) pendular; (b) funicular; (c) capillary; (d) droplet. 
where $\hat{F}$ is the normalized capillary force $\left(F_{c} / 2 \pi R \gamma\right), \hat{V}$ is the bridge volume made dimensionless by the particle radius $(R)$, and $A, B$ and $C$ are constants. In a simulation, the moisture content is assumed to be sufficiently low that bridges only form upon contact of the solid surfaces. These bridges remain in place, however, after solid contact has ceased, until the particles reach a critical separation (rupture) distance $\left(h_{c}\right)$, where $\hat{h}_{c}=\frac{h_{c}}{R}$ is given by:

$$
\hat{h}_{c}=(0.62 \theta+0.99) \hat{V}^{0.34}
$$

In order to avoid, as much as possible, system-size effects, no bridges are formed between the particles and confining walls.

\subsubsection{Viscous Forces}

Dynamic formation/breakage of liquid bridges results in a viscous force resisting motion, which may be derived from lubrication theory. It is essential that any liquid-induced cohesion model include these effects as they may become large relative to the capillary force

as the particle velocity increases [25]. In the limit of rigid spheres, Adams and Perchard [26] derive the viscous force in the normal direction $\left(F_{v_{n}}\right)$ to be:

$$
F_{v_{n}}=6 \pi \mu R v_{n_{12}} \frac{R}{2 h}
$$

where $\mu$ is the bridge fluid's viscosity, $v_{n_{12}}$ is the relative normal velocity of the approach of two spheres 1 and 2 , and $h$ is the separation between particles. In the tangential direction $\left(F_{v_{t}}\right)$, Lian et al. ${ }^{[9]}$ suggest the use of the the solution due to Goldman et al.'s ${ }^{[27]}$ for the viscous force between a sphere and a planar surface:

$$
F_{v_{t}}=\left(\frac{8}{15} \ln \frac{R}{2 h}+0.9588\right) 6 \pi \mu R v_{t_{12}}
$$

where $v_{t_{12}}$ is the relative tangential velocity of the spheres. One consideration in code development for wet systems is the surface roughness of the particles. Strictly speaking, 


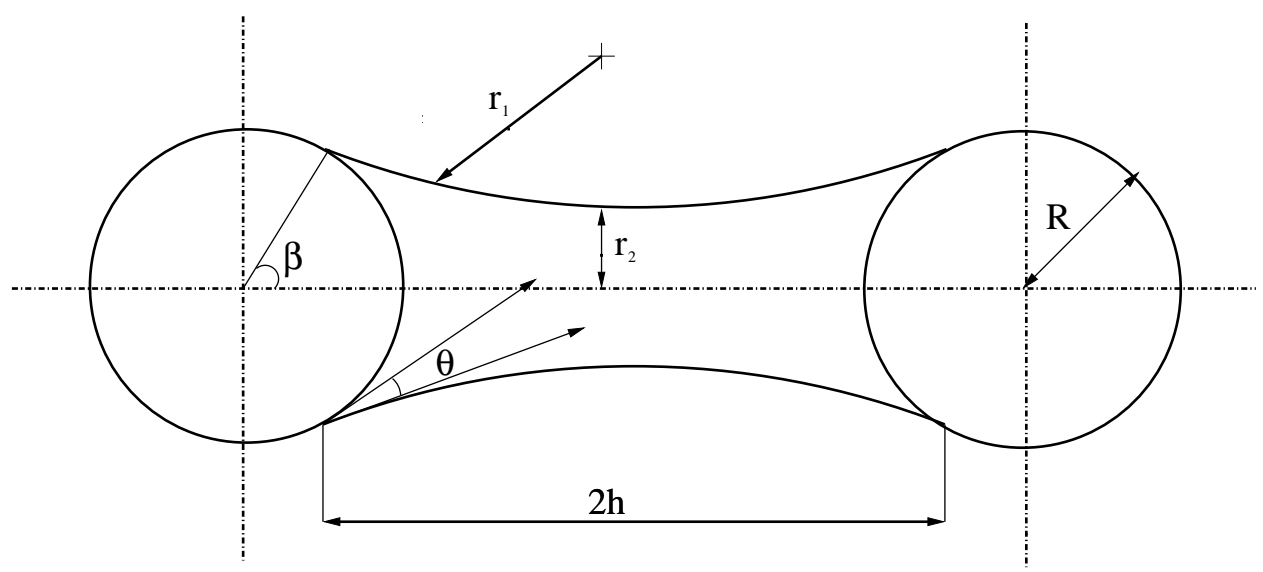

Figure 2.4 Schematic of a symmetric liquid bridge 
perfectly smooth spheres would encounter a decreasing attractive force for increasing liquid contact (while in the pendular regime), and viscous forces could become arbitrarily large for infinitely small separation distances. In practice, surface asperities limit the minimum separation distance to some constant value, $S_{o}$. In this work, $S_{o} \sim 10^{-6} \mathrm{~m}$, a reasonable value for commercial grade particles.

\subsubsection{Drag Force}

Drag between the fluidizing medium (gas) and the particle(s) couples the discrete simulation to the (continuum) fluid flow and represents the primary mode of inter-phase momentum transfer. The drag force not only depends on the local fluid flow field but also on the presence of the neighboring particles. Theoretical determination of this force is extremely difficult but semi-empirical correlations have been in place for some time ${ }^{[28]}$. In this work, we use the drag force $\left(F_{d}\right)$ suggested by Hoomans et al. ${ }^{[29]}$ as being analogous to the two-fluid implementation of Kuiper et al. [30] i.e.,

$$
F_{d}=\frac{\beta V_{p}}{1-\epsilon}\left(u-v_{p}\right)
$$

where $u$ is the local gas velocity, $v_{p}$ is the particle velocity and $\beta$ represents an inter-phase momentum exchange coefficient.

For low void fractions $(\epsilon<0.8), \beta$ is obtained from the well-known Ergun equation:

$$
\beta=150 \frac{(1-\epsilon)^{2}}{\epsilon} \frac{\mu_{g}}{2 R^{2}}+1.75(1-\epsilon) \frac{\rho_{g}}{2 R}\left(u-v_{p}\right)
$$

where $\mu_{g}$ and $\rho_{g}$ are the gas viscosity and density, respectively.

For higher void fractions $(\epsilon \geq 0.80)$, correlations presented by Wen and $\mathrm{Yu}{ }^{[31]}$, who extended the work of Richardson and Zaki ${ }^{[32]}$ are used:

$$
\beta=\frac{3}{4} C_{d} \frac{\epsilon(1-\epsilon)}{2 R} \rho_{g}\left(u-v_{p}\right) \epsilon^{-2.65}
$$


where the drag coefficient $C_{d}$ is a function of the particle Reynolds Number $\left(R e=\frac{2 R \rho_{g} \epsilon\left(u-v_{p}\right)}{\mu_{g}}\right)$

$$
C_{d}=\left\{\begin{array}{cc}
\frac{24}{R e}\left(1+0.15 R e^{0.687}\right) & R e<1000 \\
0.44 & R e \geq 1000
\end{array}\right.
$$

\subsection{Fluid Dynamics}

Anderson and Jackson ${ }^{[12]}$ formulated continuum equations representing mass and momentum balances from the point Navier-Stokes and continuity equations using the concept of local mean variables. The point variables are averaged over regions that are large compared to the particle diameter but small with respect to the characteristic dimension of the complete system. A weighting function, $g(|x-y|)$, is used in the formulation of local averages of the point variables, where $|x-y|$ denotes the separation of two arbitrary points in space. The integral of $g$ over the total space is normalized to unity:

$$
4 \pi \int_{0}^{\infty} d r=\int_{0}^{\infty} g(r) r^{2} d r
$$

The gas-phase volume-fraction at a point can be written as:

$$
\epsilon(x)_{g}=\int_{V_{g}} g(|x-y|) d V_{g}
$$

where $V_{g}$ is the fluid-phase volume. The local mean averages of the fluid-phase point properties, $<f>_{g}$, is defined by:

$$
\epsilon(x)_{g}<f>_{g}(x)=\int_{V_{g}} f(y) g(|x-y|) d V_{g} .
$$

The resulting mass and momentum balances for the fluid-phase, dropping the averaging brackets $(<>)$ on the variables are as follows:

Continuity Equation:

$$
\frac{\partial\left(\epsilon \rho_{g}\right)}{\partial t}+\left(\nabla \cdot \epsilon \rho_{g} u\right)=0
$$


Momentum Equation:

$$
\begin{aligned}
\frac{\partial\left(\epsilon \rho_{g} u\right)}{\partial t}+\left(\nabla \cdot \epsilon \rho_{g} u u\right)= & -\epsilon \nabla p-\beta\left(u-v_{p}\right)-\nabla \cdot \epsilon \tau_{g} \\
& +\epsilon \rho_{g} g
\end{aligned}
$$

where the inter-phase momentum transfer parameter, $\beta$, is obtained as in Equation 2-21 or Equation 2-22 depending upon the local particle Reynolds Number.

No-slip velocity boundary conditions are employed on the left and right walls of the fluidized bed and a Dirichlet boundary condition at the bottom with a known uniform gas inlet velocity. At the top Neumann boundary conditions are applied assuming the flow is fully developed. The outlet must be placed at a distance such that even if the bed expands, the disturbance does not reaches the top and flow remains fully developed. The pressure is fixed to a reference value at the inlet. 


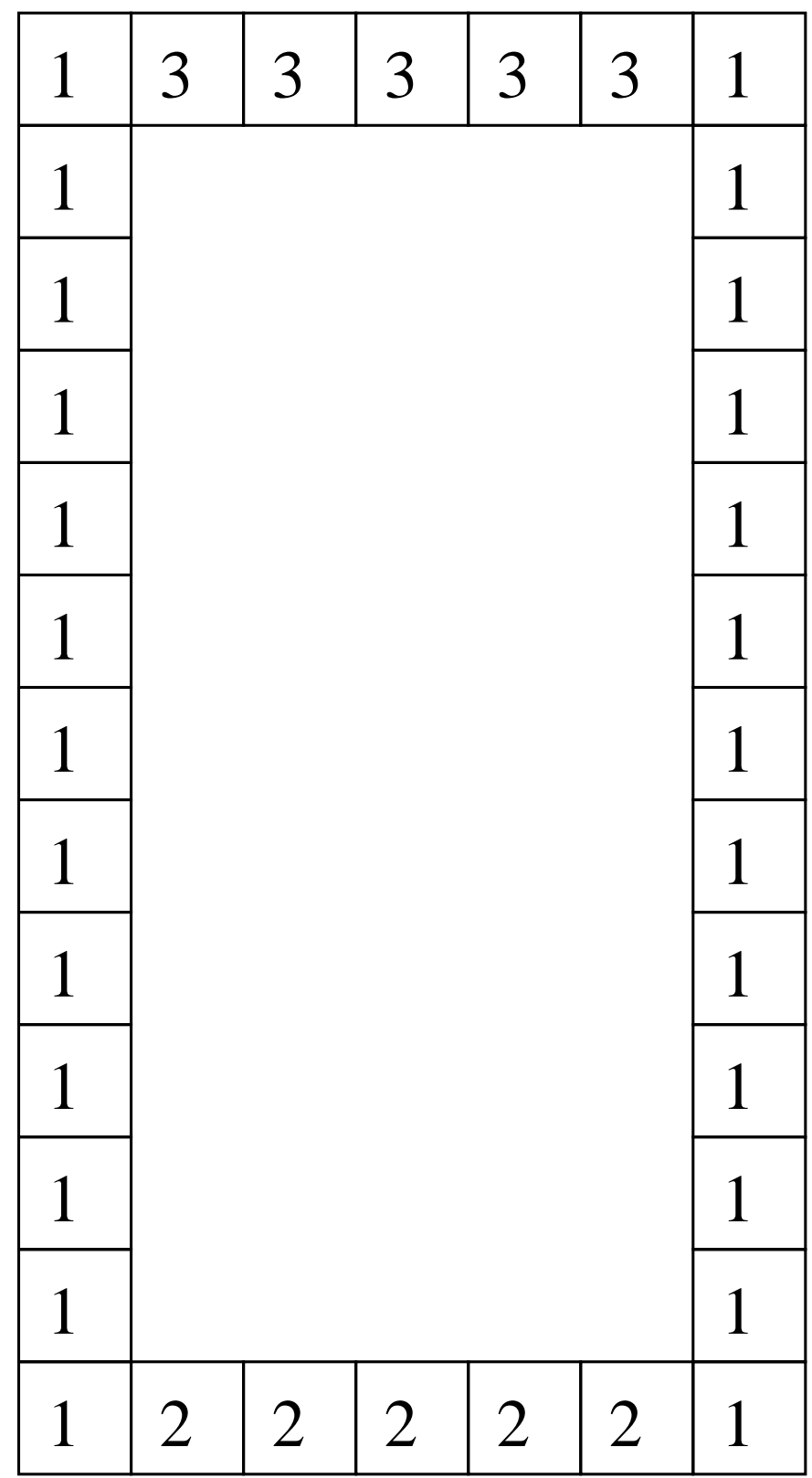

Figure 2.5 Boundary conditions used in the CFD code: [1: No-slip, 2: Specified Gasvelocity and pressure, 3: Neumann/No-flux condition] 


\subsection{CODE DEVELOPMENT}

\subsection{CFD}

CFD codes are structured around the numerical algorithms which can solve not only for

the flow and pressure field but also for the transfer of mass, heat and stress ${ }^{[33]}$. Any CFD code consists of three main parts:-

1. Pre-processor

Pre-processing mainly consists of the input of a flow problem. The input includes the definition of the geometry of the region of interest, the specification of fluid properties as well as the boundary conditions. Grid-generation is also done as part of the preprocessing.

2. Solver

The numerical methods that form the basis of the solver can be outlined as

(a) Approximation of the dependent variables using any of the differencing schemes.

(b) Discretization of the partial differential equations by using the approximation in the previous step. The commonly used numerical techniques are the finite difference, finite volume and the finite element methods.

(c) Solving for the algebraic equations.

\section{Post-processor}

Most CFD codes are equipped with data visualization tools which include the domain geometry and grid display, velocity vectors and pressure/temperature contours.

In this document the pre-processor and solver are discussed together in the later part of this chapter. 


\subsubsection{Discretization}

In our model, we use the finite volume technique for obtaining the discretized equations. The advantage of finite volume over finite differences and finite element is that conservation is enforced in the constuction of the discretized equations. The finite volume scheme begins with an integration of the governing equation over a control volume. The conservation law for the transport of a property $\phi$ can be written as:

$$
\frac{\partial}{\partial t}(\rho \phi)+\operatorname{div}(\rho u \phi)=\operatorname{div}(\tau \operatorname{grad} \phi)+S_{\phi}
$$

where $u$ represents the velocity vector and $\tau$ represents the diffusion coefficient. The first term of the equation represents the rate of change term. The second term gives the net

convective flux. The right hand side of the equation represents the net diffusive flux and the generation of property $\phi$ within the control volume. A formal integration over a control volume $\Delta V$ gives:

$$
\begin{gathered}
\int_{\Delta_{V}}\left(\int_{t}^{t+\Delta t} \frac{\partial}{\partial t}(\rho \phi) d t\right) d V+\int_{t}^{t+\Delta t}\left(\int_{A} n \cdot(\rho \mathbf{u} \phi) d A\right) d t= \\
\int_{t}^{t+\Delta t}\left(\int_{A} n \cdot(\tau \operatorname{rgad} \phi) d A\right) d t+\int_{t}^{t+\Delta t} \int_{\Delta_{V}} S_{\phi} d V d t
\end{gathered}
$$

The whole domain is subdivided into small control volumes and a staggered grid is employed to store the variables. In a staggered grid arrangement (Figure 3.1), scalar variables are stored at the nodes marked (.) and denoted by upper case alphabets. The velocities are defined at the cell faces in between the nodes denoted by lower case alphabets. In our notation $\mathrm{W}, \mathrm{E}, \mathrm{N}$ and $\mathrm{S}$ denote the nodes lying west, east, north and south of node $\mathrm{P}$ and $\mathrm{w}, \mathrm{e}, \mathrm{n}$ and $\mathrm{s}$ denote the faces lying west, east, north and south of node $\mathrm{P}$ respectively. Horizontal $(\rightarrow)$ arrows indicate the locations for $\mathrm{x}$-velocities $\left(u_{x}\right)$ and $\operatorname{vertical}(\uparrow)$ ones denote those for v-velocities $\left(u_{y}\right)$. Unrealistic oscillating pressures which might be produced by using a co-located grid are easily avoided by using a staggered grid [33,34]. In order 


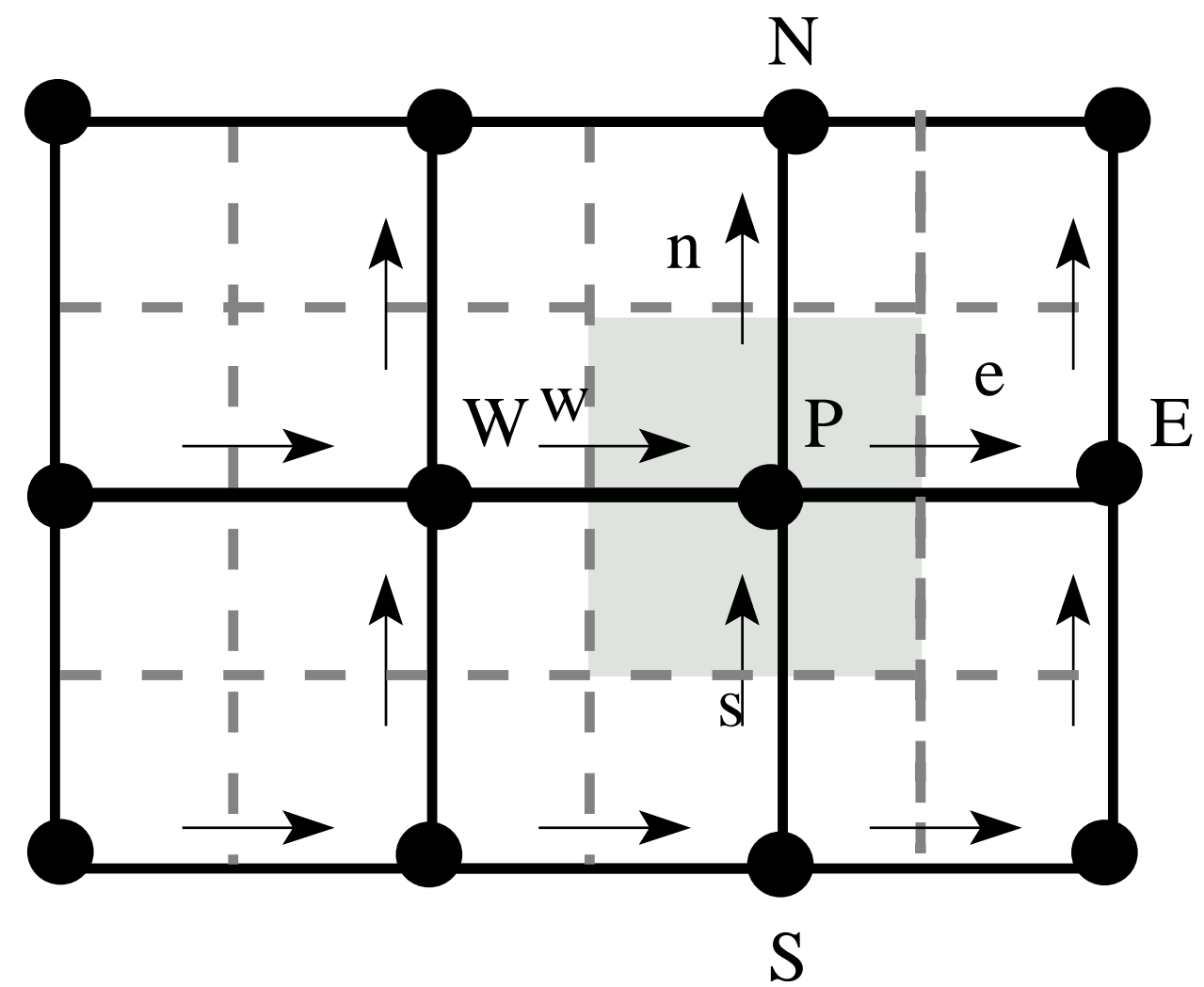

Figure 3.1 Staggered Grid arrangement where scalar variables are stored at the nodes marked $(\cdot)$ and the velocities are defined at the cell faces in between the nodes. 
to derive useful forms of the discretized equation, an approximation of the diffusive and convective terms is needed. Here the central differencing scheme is used to approximate the diffusive terms $\left(\tau A \frac{\partial \phi}{\partial x}\right)$. For a uniform grid, an expression for the value of a property at a face can be written as:

$$
\begin{gathered}
\left(\tau A \frac{\partial \phi}{\partial x}\right)_{e}=\tau_{e} A_{e}\left(\frac{\phi_{E}-\phi_{P}}{\delta x_{P E}}\right) \\
\left(\tau A \frac{\partial \phi}{\partial x}\right)_{w}=\tau_{w} A_{w}\left(\frac{\phi_{P}-\phi_{W}}{\delta x_{W P}}\right)
\end{gathered}
$$

where $\delta x_{P E}$ and $\delta x_{W P}$ represent the distances between points $P$ and $E$ and $W$ and $P$ respectively, $A_{e}$ and $A_{w}$ represent the face areas, $\tau_{e}$ and $\tau_{w}$ represent the diffusivity at the faces. The central differencing is a direct outcome of the Taylor-Series formulation.

For the convective terms $(\rho u \phi)$, using the central differencing scheme results in the possibility of negative discretized coefficients which can lead to physically unrealistic results. One method of avoiding this difficulty is to use the upwind scheme. According to the upwind scheme, the value of $\phi$ at an interface is equal to the value of $\phi$ at the grid point on the upwind side. In other words,

$$
\begin{gathered}
\phi_{e}=\phi_{P} \text { if } F_{e} \geq 0 \\
\phi_{e}=\phi_{W} \text { if } F_{e} \leq 0
\end{gathered}
$$

where $F_{e}=\left(\rho u_{x}\right)_{e}$.

The conditional statements can be re-written as:

$$
F_{e} \phi_{e}=\phi_{P}\left\|F_{e}, 0\right\|-\phi_{E}\left\|-F_{e}, 0\right\|
$$

where $\|A, B\|$ represents the maximum of $\mathrm{A}$ and $\mathrm{B}$.

A fully implicit scheme is adopted for discretizing the temporal terms, where the variable assumes the new value at the beginning of the time step. With the implicit time scheme, 
all flux coefficients are positive making it stable and robust for any size of time step. Any discretized equation for a property $\phi$ at a point $p$ then has the following form:

$$
a_{p} \phi_{p}=\Sigma a_{n b} \phi_{n b}+b
$$

where the subscript $n b$ denotes a neighbor point, a represents the discretised flux at the point $p$ and $b$ represents the source term.

Any given discretized equation should have the following properties:

1. Conservativeness: To ensure conservation of $\phi$ for the whole solution domain the flux of $\phi$ leaving a control volume across a certain face must be equal to the flux of $\phi$ entering the adjacent control volume through the same face. To achieve this the flux through a common face must be represented by the same expression.

2. Boundedness: The 'boundedness' criterion states that in the absence of sources, the internal nodal values of property $\phi$, should be bounded by its boundary values. To ensure this the following should be kept in mind:

- All coefficients of the discretized equation should have the same sign.

- The Scarborough criterion must be satisfied.

$$
\Sigma \frac{a_{n b}}{a_{P}}\left\{\begin{array}{l}
\leq 1 \\
<1
\end{array} \quad\right. \text { at one node at least }
$$

The final two-dimensional discretized equation for a property $\phi$ can be written as:

$$
a_{p} \phi_{p}=a_{E} \phi_{E}+a_{W} \phi_{W}+a_{N} \phi_{N}+a_{S} \phi_{S}+b
$$

where,

$$
\begin{aligned}
& a_{E}=\tau_{e} A_{e}+\left\|-F_{e}, 0\right\| \\
& a_{W}=\tau_{w} A_{w}+\left\|F_{w}, 0\right\|
\end{aligned}
$$




$$
\begin{gathered}
a_{N}=\tau_{n} A_{n}+\left\|-F_{n}, 0\right\| \\
a_{S}=\tau_{s} A_{s}+\left\|F_{s}, 0\right\| \\
a_{P}^{o}=\frac{\rho_{P}^{o} \delta x \delta y}{\delta t} \\
b=S_{C}+a_{P}^{o} \phi_{P}^{o} \\
a_{P}=a_{E}+a_{W}+a_{N}+a_{S}+a_{P}^{o}-S_{P} \delta x \delta y
\end{gathered}
$$

where $\delta x$ and $\delta y$ represent the dimensions of the control volume. $\phi_{P}^{o}$ and $\rho_{P}^{o}$ refer to the values at the previous time step.

\subsubsection{SIMPLE}

A discretized equation for pressure is needed for solving the pressure-velocity field. If the flow is compressible, the continuity euqation is used as a transport equation for density. However for incompressible flows the density is constant and not linked to the pressure. The continuity equation is used to derive an equation for pressure but it introduces a constraint on the solution of the flow field: if the correct pressure field is applied in the momentum equations the resulting velocity field should satisfy continuity. The SIMPLE (Semi-Implicit Pressure Linked Equations) algorithm ${ }^{[34]}$ tackles these problems by adopting an iterative solution strategy. The pressure and velocities are resolved into two components, guessed and corrected:

$$
\begin{gathered}
p=p^{*}+p^{\prime} \\
u_{x}=u_{x}^{*}+u_{x}^{\prime}
\end{gathered}
$$




$$
u_{y}=u_{y}^{*}+u_{y}^{\prime}
$$

where superscript $*$ denotes the guessed part and ' denotes the corrected portion. The momentum equation for $\mathrm{x}$-velocity can be written by replacing $\phi$ with the $u_{x}$ in the Equation 3-9:

$$
a_{e} u_{x_{e}}=\Sigma a_{n b} u_{x_{n b}}+\left(p_{P}-p_{E}\right) A_{e}+b
$$

where subscript $n b$ represents the neighbor coefficients.

The momentum equation for y-velocity can be written as:

$$
a_{n} u_{y_{n}}=\Sigma a_{n b} u_{y_{n b}}+\left(p_{P}-p_{N}\right) A_{n}+b
$$

Using Equation 3-17 and Equation 3-18, the x-momentum equation can be rewritten as:

$$
\begin{gathered}
a_{e} u_{x_{e}}^{*}=\Sigma a_{n b} u_{x_{n b}}^{*}+\left(p_{P}^{*}-p_{E}^{*}\right) A_{e}+b \\
a_{e} u_{x_{e}}^{\prime}=\Sigma a_{n b} u_{x_{n b}}^{\prime}+\left(p_{P}^{\prime}-p_{E}^{\prime}\right) A_{e} .
\end{gathered}
$$

Similarly the y-momentum equation can be rewritten as:

$$
\begin{gathered}
a_{n} u_{y_{n}}^{*}=\Sigma a_{n b} u_{y_{n b}}^{*}+\left(p_{P}^{*}-p_{N}^{*}\right) A_{n}+b \\
a_{n} u_{y_{n}}^{\prime}=\Sigma a_{n b} u_{y_{n b}}^{\prime}+\left(p_{P}^{\prime}-p_{N}^{\prime}\right) A_{n} .
\end{gathered}
$$

If the term $\Sigma a_{n b} u_{n b}^{\prime}$ is dropped from the Equation 3-23, the equation can be rewritten as:

$$
u_{x_{e}}^{\prime}=d_{e}\left(p_{P}^{\prime}-p_{E}^{\prime}\right) A_{e}
$$

where $d_{e}=\frac{A_{e}}{a_{e}}$.

Hence, the total velocity can be written as:

$$
u_{x_{e}}=u_{x_{e}}^{*}+d_{e}\left(p_{P}^{\prime}-p_{E}^{\prime}\right) .
$$


A similar procedure can be applied to the y-velocities to obtain:

$$
u_{y_{n}}=u_{y_{n}}^{*}+d_{n}\left(p_{P}^{\prime}-p_{N}^{\prime}\right)
$$

These $\mathrm{x}$ and $\mathrm{y}$ velocities can be plugged into the continuity equation and a pressure correction equation can be obtained. The continuity equation for an incompressible flow can be written as:

$$
\frac{\partial u_{x}}{\partial x}+\frac{\partial u_{y}}{\partial y}=0
$$

The corresponding discretized equation becomes:

$$
a_{P} P_{P}^{\prime}=a_{E} P_{E}^{\prime}+a_{W} P_{W}^{\prime}+a_{N} P_{N}^{\prime}+a_{S} P_{S}^{\prime}+b^{\prime}
$$

where,

$$
\begin{gathered}
a_{E}=\rho_{e} d_{e} A_{e} \\
a_{W}=\rho_{w} d_{w} A_{w} \\
a_{N}=\rho_{n} d_{n} A_{n} \\
a_{S}=\rho_{s} d_{s} A_{s} \\
a_{P}=a_{E}+a_{W}+a_{N}+a_{S} ; \\
b^{\prime}=\left[\left(\rho_{w} u_{x_{w}}^{*} A_{w}\right)-\left(\rho_{e} u_{x_{e}}^{*} A_{e}\right)\right]+\left[\left(\rho_{s} u_{y_{s}}^{*} A_{s}\right)-\left(\rho_{s} u_{y_{n}}^{*} A_{n}\right)\right] .
\end{gathered}
$$

The sequence of the operations in the SIMPLE algorithm is as follows:

1. Guess the pressure field $p^{*}$ 
2. Solve the momentum Equation 3-22

3. Solve the $p^{\prime}$ Equation 3-30

4. Calculate the total $p$ by adding $p$ to $p^{*}$

5. Calculate $u_{x}, u_{y}$ from their starred values using the velocity-correction formulas: Equation 3-27 and Equation 3-28

6. Treat the corrected pressure $p$ as a new guessed pressure and repeat the above steps until a converged solution is obtained.

The solver used in the code was the Gauss-Seidel step-by-step method. The values of the variable are calculated by visiting each grid point sequentially. Only one set of variables are maintained in the memory. For the first iteration these represent initial guesses. For neighbors that have already been visited during the current iteration, the new values are used. When all grid points have been visited, one iteration of Gauss-Seidel is over.

\subsection{Particle Dynamics}

Like any Lagrangian simulation, PD is computationally intensive. A considerable amount of time is spent in the contact detection. To overcome this issue we implemented the No Binary Search algorithm as put forward by Munjiza et al. ${ }^{[35]}$. The NBS contact detection is based on space decomposition. The space is divided into identical square cells of size $2 R$ where $\mathrm{R}$ is the radius of the largest particle. Mapping of a particle is defined in such a way that each particle is assigned to one and only one cell. A particle with center coordinates $(\mathrm{x}, \mathrm{y}, \mathrm{z})$ is assigned to the cell $(i x, i y, i z)$, where $i x$ and $i y$ are integerized co-ordinates of the particle. For example, in Figure 3.2, particle 5 would have integerized coordinates of $(1,3,0)$. 


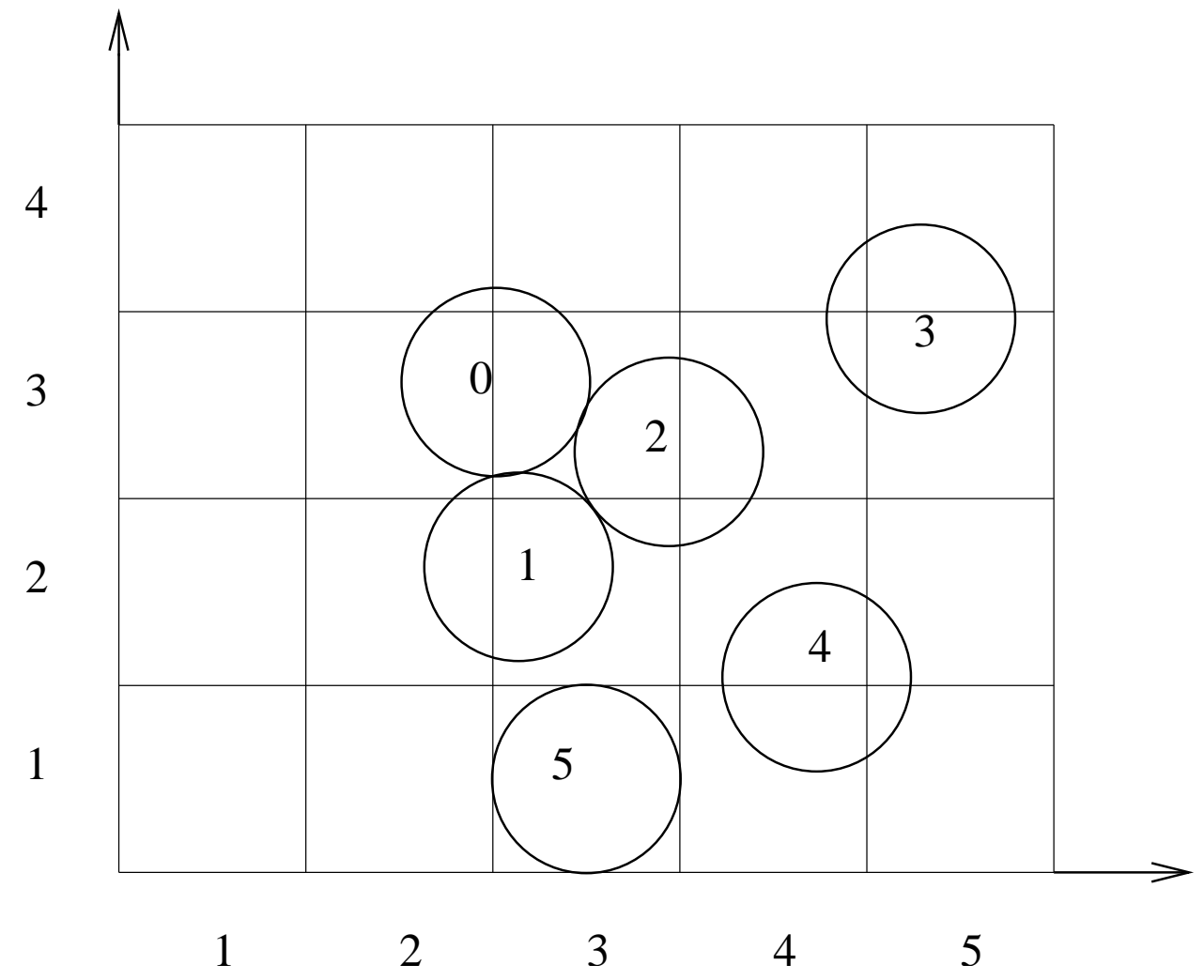

Figure 3.2 NBS Space decomposition. The cell size is equal to the largest particle diameter. 
The sequence of operations in a NBS is as follows:

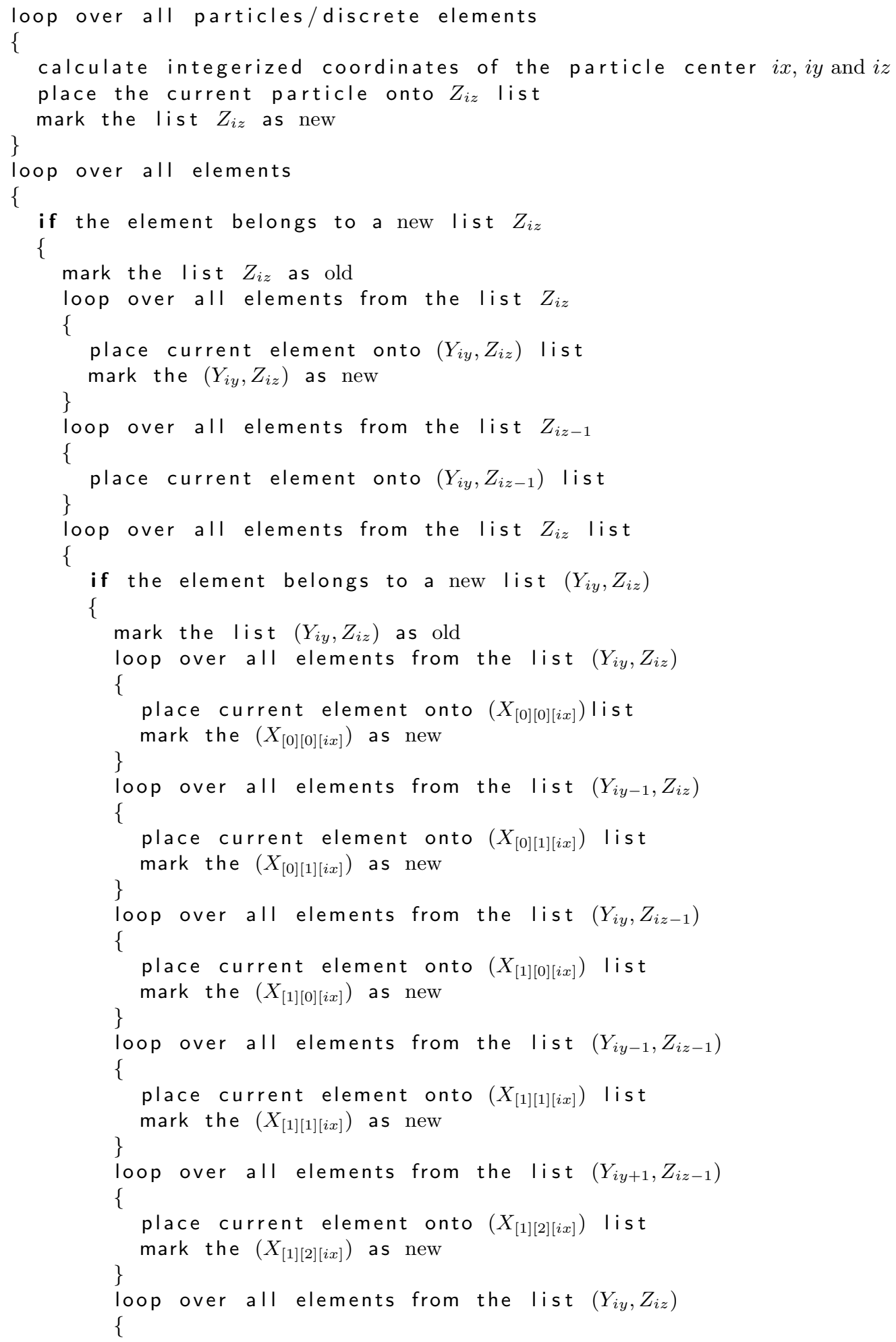




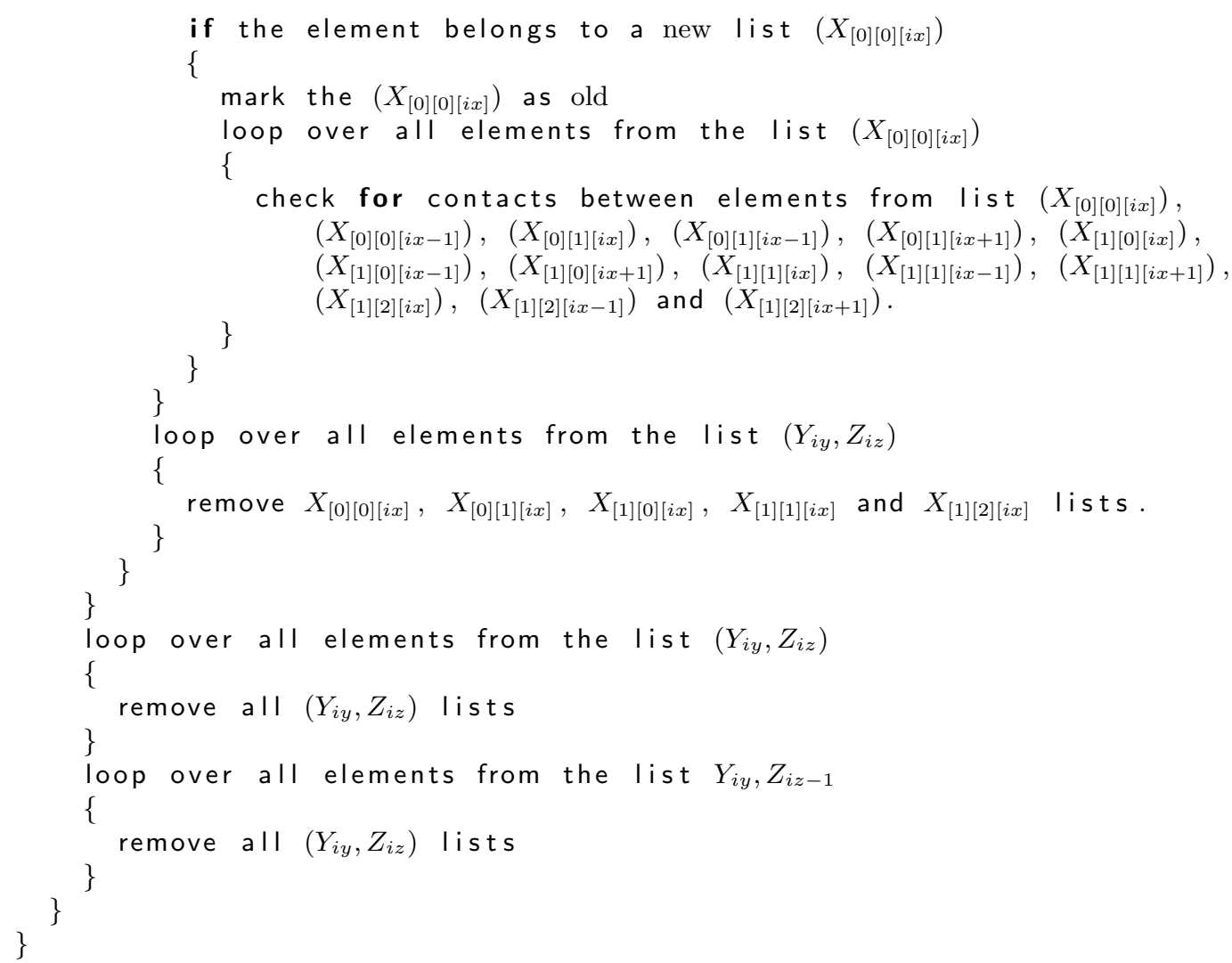

The NBS algorithm works equally well for both dense and loose packing since contact detection is performed for only those cells which have particles linked to them. Also, a separate sorting step is not required in this method. These characteristics often yield improved performance for NBS. The algorithm is compared with conventional brute force and binary search algorithms in Figure 3.3 and is seen to be much more efficient. However, a limitation of NBS is that this improvement is seen only when the particles are of comparable sizes.

Since the grid sizes of the particle phase and fluid phase are different, properties of fluid phase (velocity, void-fraction) are calculated on a local basis. The particles are first mapped on to the Eulerian grid and the local properties are then calculated by taking a weighted contribution. 


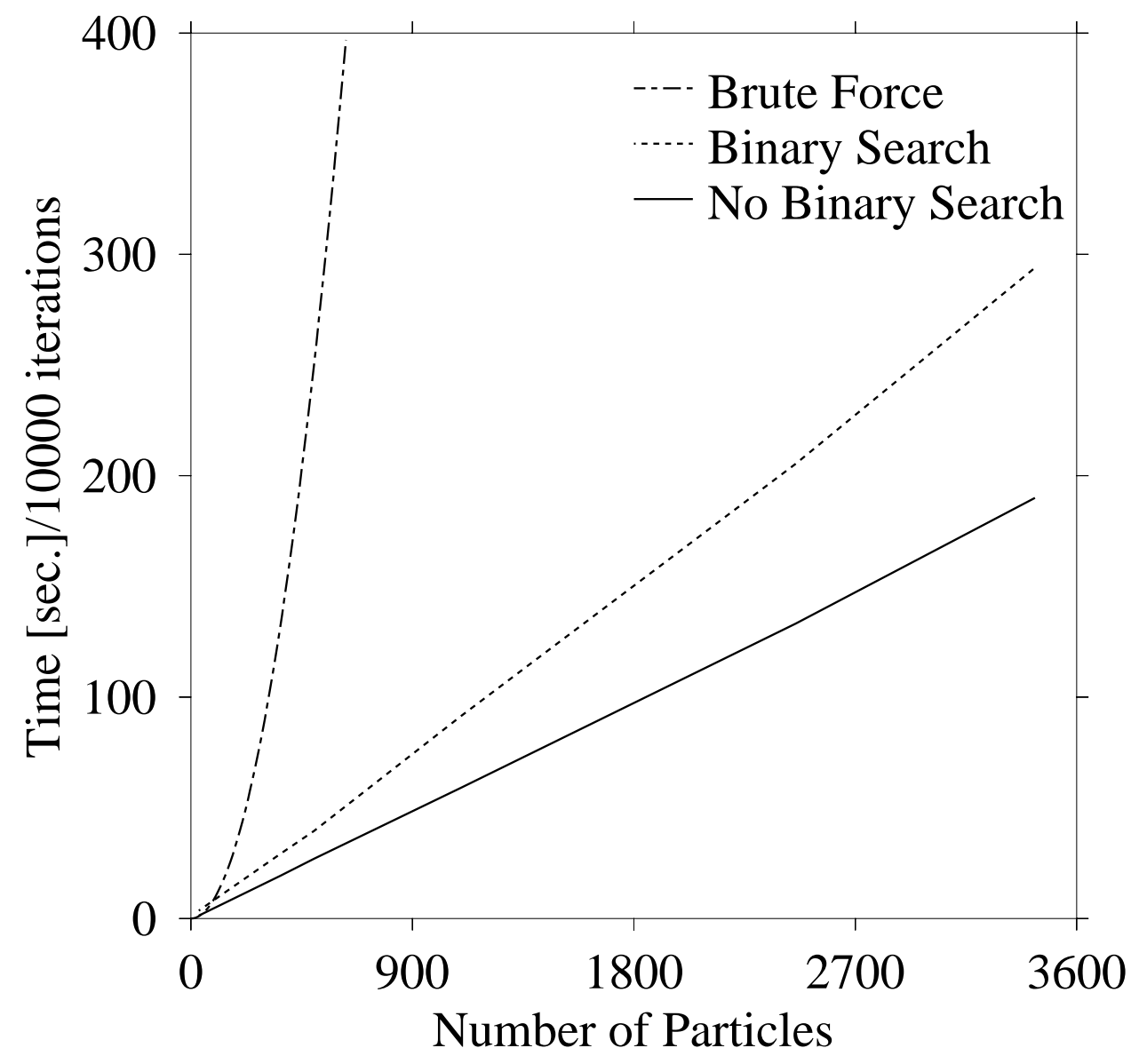

Figure 3.3 Comparison of contact-detection algorithms. 


\subsection{CHARACTERIZATION TOOLS}

\subsection{Granular Capillary Number}

The relevant variables that need to be considered in studying gas-solid flows include $\left(R, \rho_{s}, \rho_{g}, g, \gamma, \hat{V}, g, \delta, \mu_{g}\right)$, where $\delta$ is a height of the bed and $\hat{V}$ is the relative velocity of the particle with respect to the fluid velocity $\left(\hat{V}=u-v_{p}\right)$. It should be noted that, in this study, the viscosity of the liquid bridge fluid, $\mu$, is maintained constant as the effects of dynamic viscous forces in wet media have been aptly explored by Ennis et al. ${ }^{[36,25]}$. By a Buckingham Pi analysis, five dimensionless groups are determined:

$$
\phi_{1}=\frac{\delta}{R}, \phi_{2}=\frac{\rho_{s}}{\rho_{g}}, \phi_{3}=\frac{\gamma}{\rho_{s} g R^{2}}, \phi_{4}=\frac{\hat{V}^{2}}{g R}, \phi_{5}=\frac{\gamma}{\mu_{g} \hat{V}}
$$

The trivial dimensionless groups arising from the density and length-scale ratios do not directly factor into studying cohesion and are ignored. The remaining three may be thought of as combinations of forces acting in the system: the cohesive force, the force due to particle collisions, the weight of a particle and the drag force. Previous work by Nase et al. [4] detail the significance of the third and fourth group of variables for characterization of wet granular systems, so they are only briefly reviewed below.

The third group, the Granular Bond Number ${ }^{[4]}\left(B o_{g}\right)$, represents the ratio of the maximum capillary force to the weight of a particle. This group is dominant in characterizing the effects of cohesion in static or near-static systems. Figure 4.1 shows that, while essentially no cohesive effects are present at $B o_{g}<1$, a dramatic transition in the slope of a static pile is evidenced as the $B o_{g}$ is increased above unity. The fourth group along with the $B o_{g}$ and the first group represents the ratio of maximum cohesive force to the collisional force due to Bagnold ${ }^{[37]}$ and is called the Collision Number ${ }^{[4]}(\mathrm{Co})$. This number becomes dominant in highly sheared granular materials where $B o_{g}>1$. 


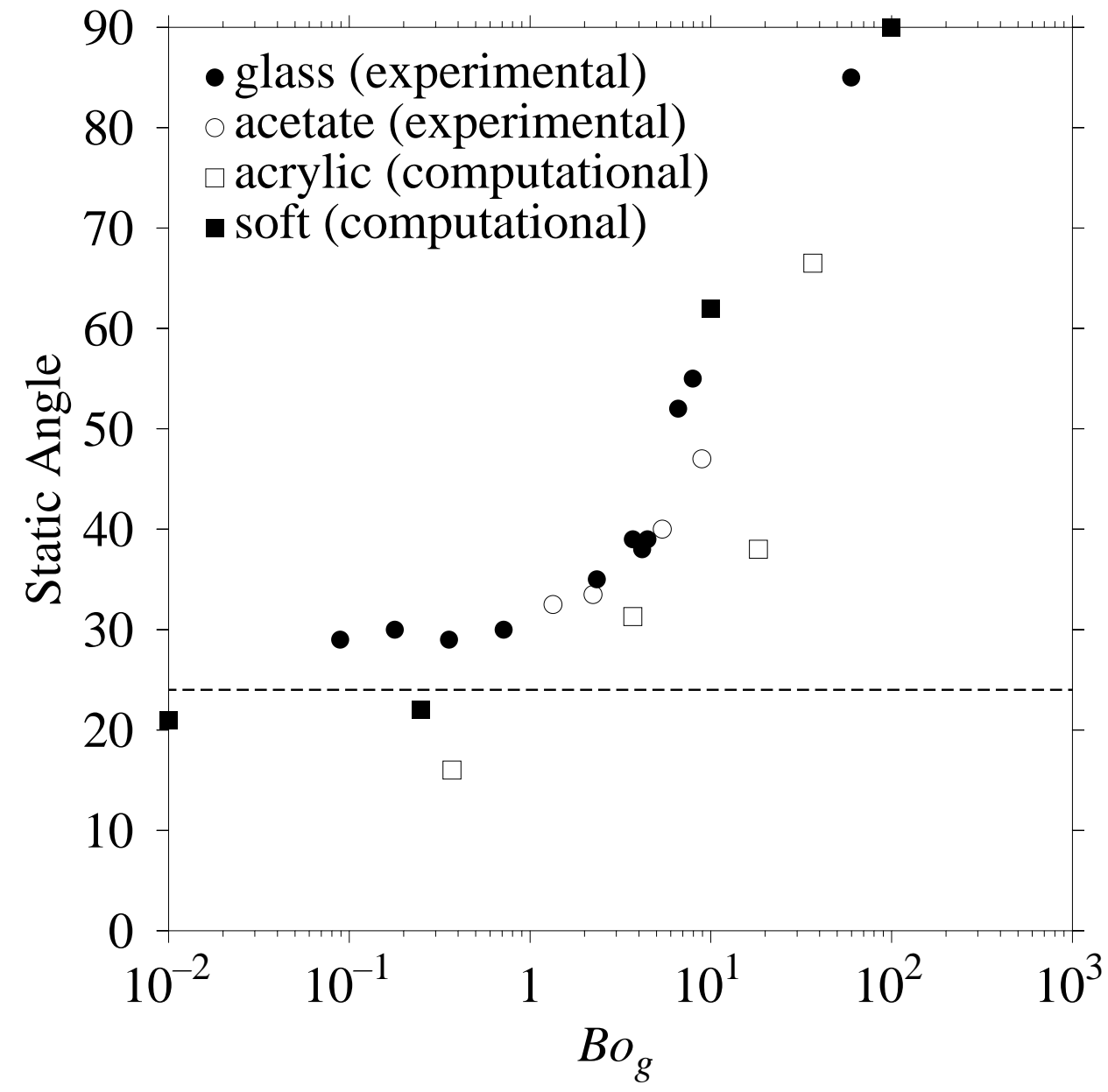

Figure 4.1 Characterization via the Granular Bond Number (Nase et al. ${ }^{[4]}$ ). 
Figure 4.2 shows how the difference between the dynamic angle of repose of wet versus dry material changes with increasing Co. A marked decrease in cohesive effects is observed as shearing forces are increased, even in cases where $B o_{g}>1$. The fifth group, newly introduced in this work, can be thought of as the ratio of the cohesive force to the drag force. For $R e<10$, an easily interpreted dimensionless number can be obtained directly from this fifth group. The drag force on particles can be derived from the Carman-Kozeny equation as:

$$
F_{d}=60 \mu_{g}\left(u-v_{p}\right) R \frac{\left(1-\epsilon^{2}\right)}{\epsilon^{3}}
$$

which may be combined with an expression for the maximum capillary force:

$$
F_{c}=2 \pi R \gamma
$$

to yield the Granular Capillary Number as:

$$
C a_{g}=\frac{F_{c}}{F_{d}}=\frac{\epsilon^{3} \gamma}{30(1-\epsilon)^{2} \mu_{g}\left(u-v_{p}\right)}
$$

For $R e>2000$, the fluid drag on the particle is no longer linearly proportional to the velocity difference, instead $\frac{\phi_{2} \phi_{3}}{\phi_{4}}$ can be used to represent the ratio of the cohesive to the drag force. The drag force on the particle can be derived from the Burke-Plummer equation as:

$$
F_{d}=\frac{7}{6} \rho_{g}\left(u-v_{p}\right)^{2} R^{2} \frac{(1-\epsilon)}{\epsilon^{3}}
$$

which yields:

$$
C a_{g}=\frac{12 \epsilon^{3} \gamma}{7(1-\epsilon) \rho_{g}\left(u-v_{p}\right)^{2} R}
$$

A general equation for the drag force $\left(C a_{g}\right)$ can be written using the Ergun's equation:

$$
F_{d}=50 \mu_{g}\left(u-v_{p}\right) R \frac{\left(1-\epsilon^{2}\right)}{\epsilon^{3}}+\frac{7}{6} \rho_{g}\left(u-v_{p}\right)^{2} R^{2} \frac{(1-\epsilon)}{\epsilon^{3}}
$$




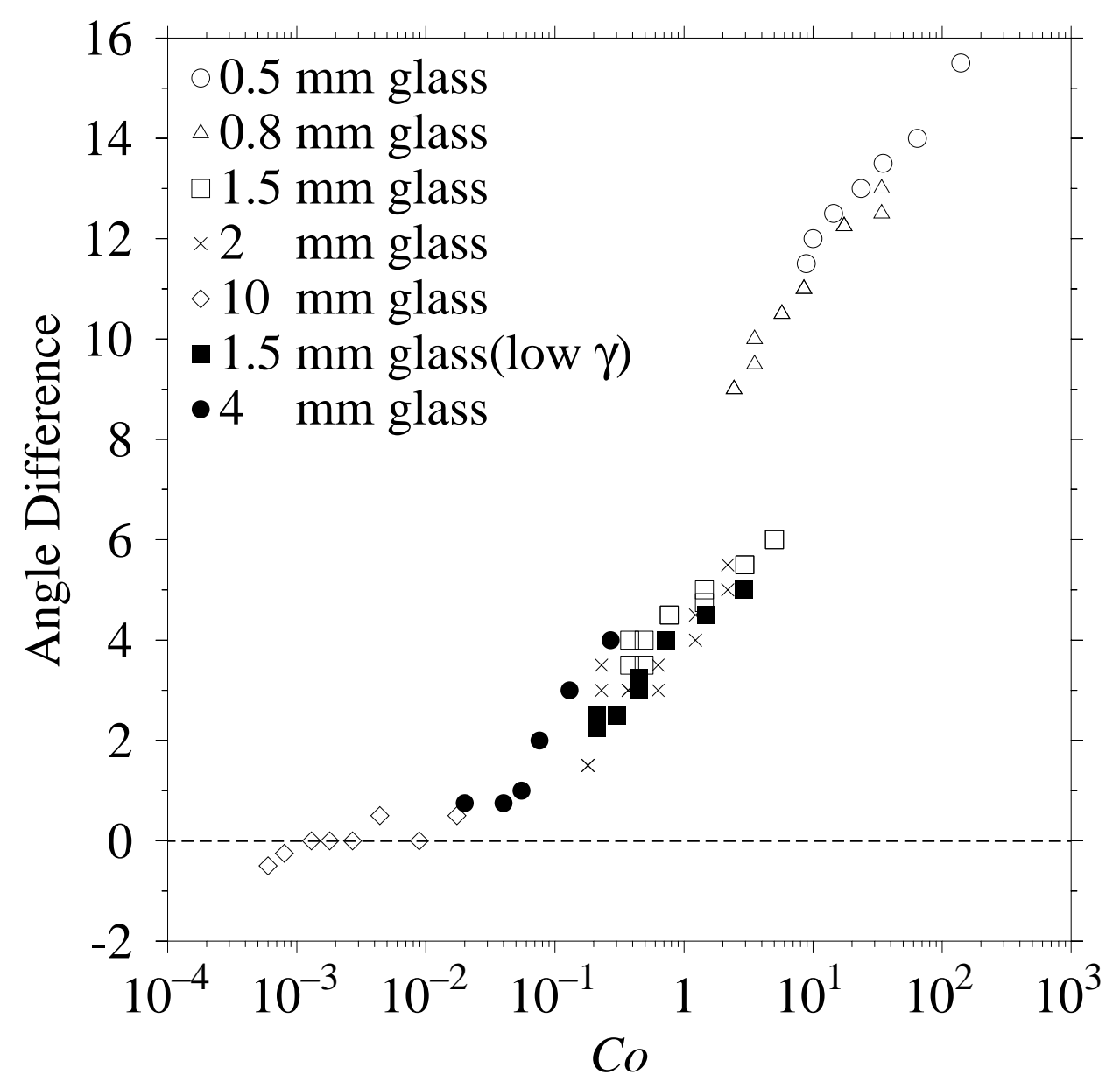

Figure 4.2 Characterization via the Collision Number (Nase et al. ${ }^{[4]}$ ). 
which yields a completely general form of the Granular Capillary Number $\left(C a_{g}\right)$

$$
C a_{g}=\frac{12 \epsilon^{3} \gamma}{(1-\epsilon)\left(u-v_{p}\right)}\left[\frac{1}{300(1-\epsilon) \mu_{g}+7 \rho_{g}\left(u-v_{p}\right) R}\right]
$$

It is important to note that, when using our discrete characterization tools, all relevant dimensionless groups must be greater than unity in order for a granular material to be considered "cohesive". Moreover, it is the smallest of the dimensionless groups that then serves as the mitigating factor with respect to cohesion. Therefore, we will examine systems where both $B o_{g}$ and $C o$ and larger than 1 and we will vary (primarily) $C a_{g}$.

\subsection{Applications}

Computer simulations are run using soda lime glass particles and air with properties as given in Table 4.1. The initial conditions are created by letting the particles fall freely under gravity from a randomly perturbed rectangular lattice. Dimensions of the bed are $30 \mathrm{~mm}$ (width $) \times 510 \mathrm{~mm}$ (height $) \times 1 \mathrm{~mm}$ (thickness $)$. Each simulation is initially run with

dry particles in order to get baseline values for comparison to differing degrees of cohesion. In the cohesive simulations, the $C a_{g}$ is varied by changing the surface tension of the liquid bridge fluid (as might be done with surfactants, experimentally), while maintaining its viscosity as a constant.

Table 4.1 Parameters used in the simulation

\begin{tabular}{|l|l|}
\hline Parameter & Soda-Lime Glass \\
\hline Density & $2600 \mathrm{~kg} / \mathrm{m}^{3}$ \\
\hline Poisson ratio & 0.25 \\
\hline Young's Modulus & $66 \mathrm{GPa}$ \\
\hline Particle radius & $5.0 \times 10^{-4} \mathrm{~m}$ \\
\hline Friction coefficient & 0.29 \\
\hline Parameter & Air \\
\hline Fluid Density & $1.23 \mathrm{~kg} / \mathrm{m}^{3}$ \\
\hline Viscosity & $1.80 \times 10^{-5} \mathrm{~kg} / \mathrm{ms}$ \\
\hline
\end{tabular}




\section{DRY}
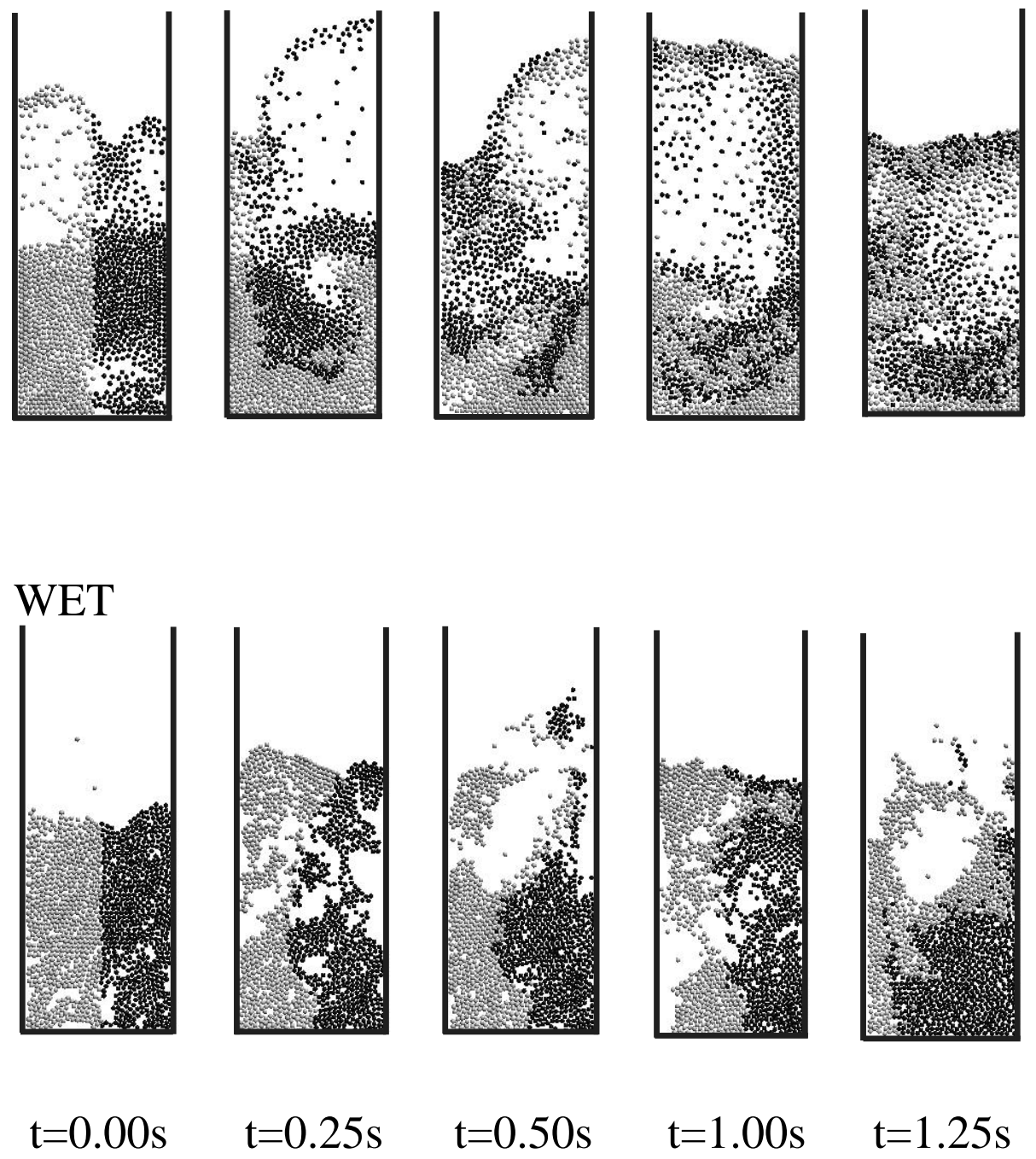

$\mathrm{t}=1.00 \mathrm{~s}$

$$
\mathrm{t}=1.25 \mathrm{~s}
$$

Figure 4.3 Mixing progress for Cohesive/Non-Cohesive materials. 


\subsubsection{Mixing}

The mixing in gas-solid systems is often extremely rapid compared to mixing in surfacedominated flows ${ }^{[38]}$. Powder mixing in a gas-solid flow predominantly occurs by convective mixing. Convective mixing occurs by deliberate movement of packets of particles around the mixture. These packets break down into smaller clumps and continue to displace relative to each other. This proceeds while the clumps become smaller and smaller [39]. The reduction in size of clumps with time depends on the extent of cohesion amongst the solids. In this Section we examine changes of the mixing rate of mechanically identical particles with changes in gas velocity as well as liquid bridge surface tension. In all cases, the system is assumed to be completely segregated initially with the right-half of the bed consisting of one type (color) of particle and the left-half another. Typical snapshots of a dry and wet simulation can be seen in Figure 4.3. We measure the intensity of segregation, essentially the standard deviation of the local concentration, where we define the local concentration to consist of each particle's 20 nearest neighbors. If the intensity of segregation is plotted as a function of time, the value - initially at 0.5 for completely segregated - will decrease as the system proceeds toward a completely mixed state. Figure 4.4 shows the evolution of the intensity of segregation at several different gas velocities, while Figure 4.5 shows similar results for a fixed gas velocity and varying liquid bridge surface tension. By fitting these data to an exponential function, a mixing rate constant can be extracted.

Examining both Figure 4.4 and Figure 4.5, it is clear that higher velocities and/or lower surface tensions result in larger (faster) mixing rates. Our definition of the Granular Capillary Number $\left(C a_{g}\right)$, then suggests that the importance of cohesion to mixing is determined by an interplay between the capillary force and the fluid drag. Plotting the resultant mixing rate constants as a function of $C a_{g}$, in Figure 4.6, shows that this assertion is valid. Mixing rates are high for $C a_{g}<1$ and drop dramatically when $C a_{g}>1$. 


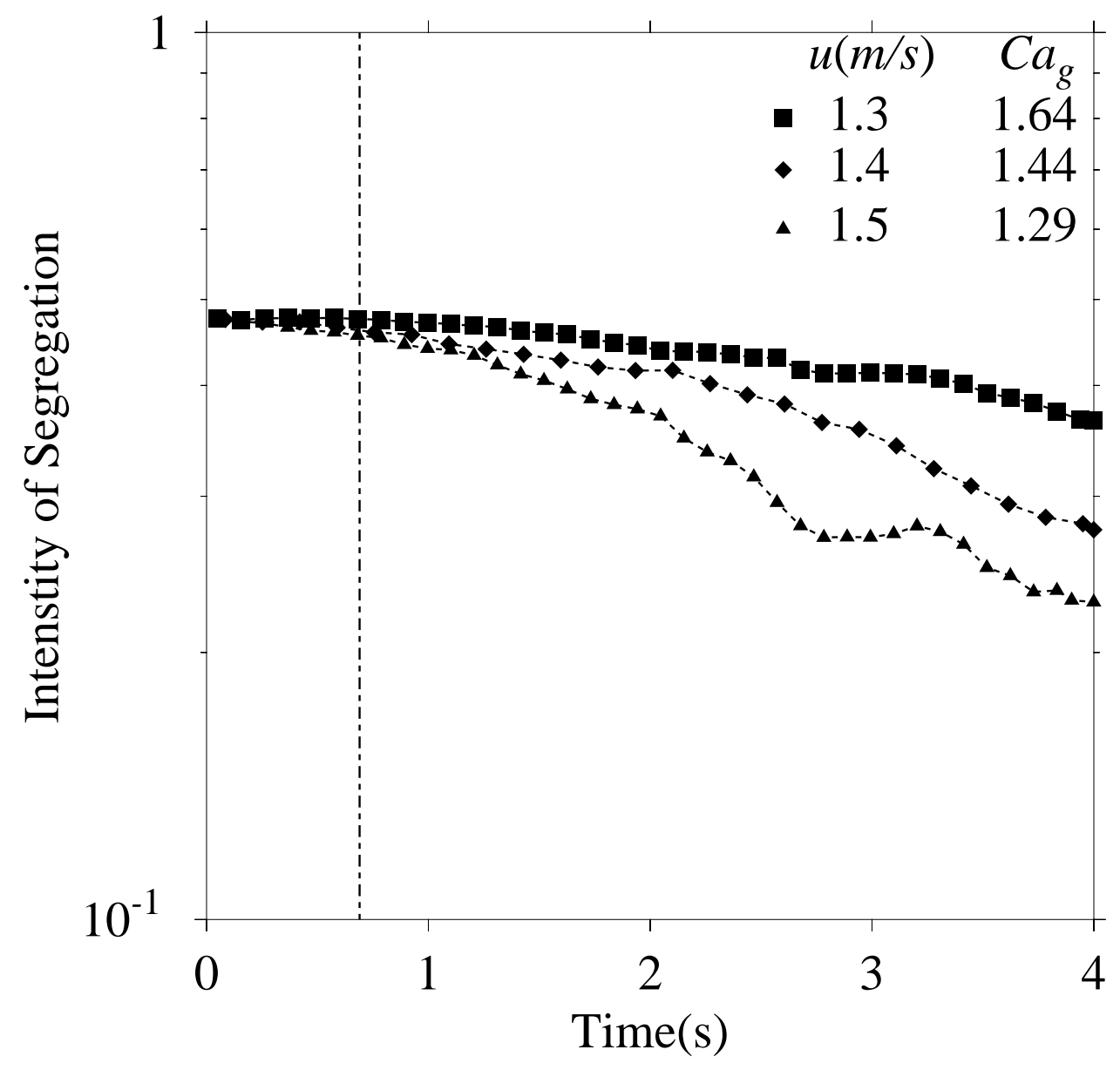

Figure 4.4 Mixing Rates at different gas velocities. 


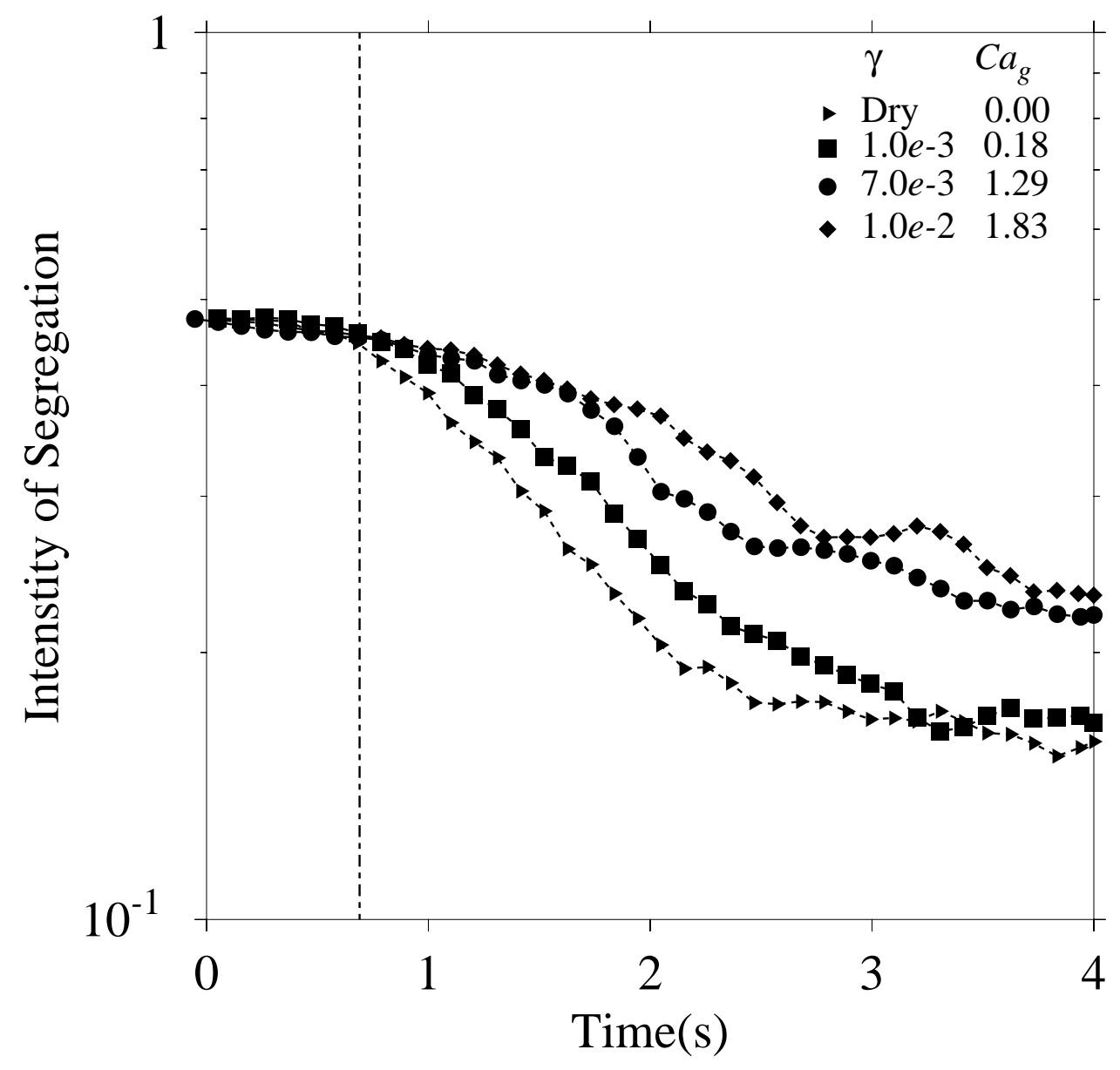

Figure 4.5 Mixing Rates at different surface tensions. 


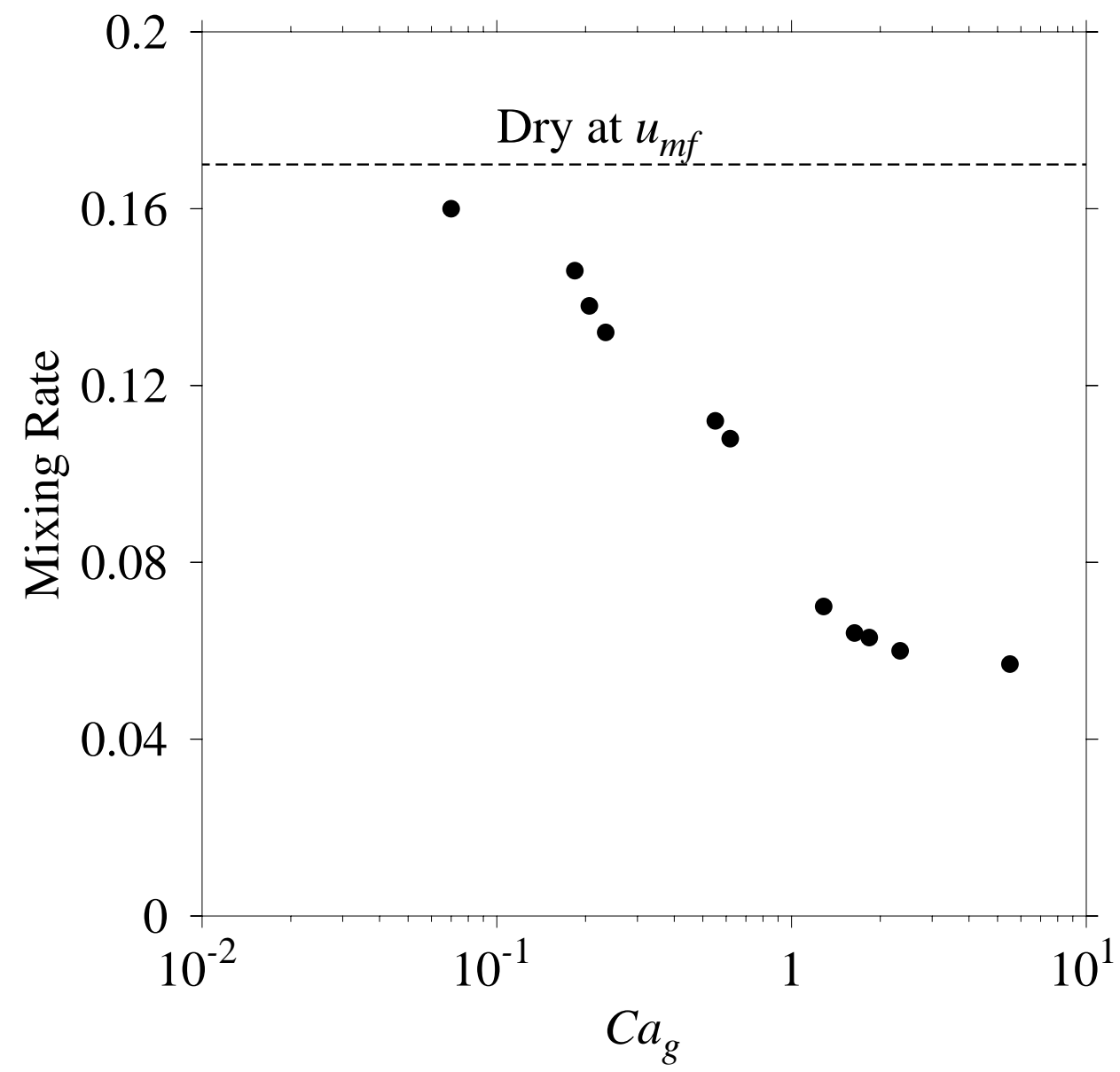

Figure 4.6 Mixing Rate versus Granular Capillary Number 


\subsubsection{Fluidization}

Perhaps a more practical test of the utility of the Granular Capillary Number is for quantifying the onset of fluidization in gas-solid systems. The minimum fluidization velocity $\left(u_{m f}\right)$ is typically defined as the velocity at which the bed pressure drop goes through a maximum value. A critical component of this definition is that, while the pressure drop is ultimately determined solely by the weight of the fluidized particles, the value of the pressure drop can exceed this limit prior/after to fluidization. In the small fluidization systems examined here a simpler, but equivalent, definition of the minimum fluidization velocity is used. The approach used for determining the minimum fluidization velocity is similar in spirit to that followed by Kafui et al. ${ }^{[40]}$ which is based on monitoring the state of the particle connectivity network. Figure 4.7 shows a plot of pressure drop versus time for different gas-velocities with a traditional time-averaged pressure drop $(\overline{\Delta P})$ versus $u_{m f}$. For $u<u_{m f}$, the pressure drop essentially remains constant, and for $u \geq u_{m f}$, the pressure drop varies with time. The amplitude and standard deviation of the pressure disturbance also increase with an increase in the fluidization velocity. Hence, the minimum fluidization velocity can be defined as the velocity at which the magnitude of fluctuations (standard deviation) of the pressure drop goes through a step change (see Figure 4.8). This technique provides for more reproducible results and avoids difficulties in averaging for small systems.

We find that, with an increase in the $C a_{g}$ (surface tension), the velocity necessary to achieve a fluidized system increases from that of the completely dry (non-cohesive) case. Figure 4.9 shows a plot of the percentage increase in the minimum fluidization velocity as a function of the $C a_{g}$. For values of surface tension where the $C a_{g}<1$, changes in the fluidization velocity from that of a completely dry granular material are essentially unmeasurable; however, for larger surface tensions, where the values of $C a_{g}>1$, the minimum 


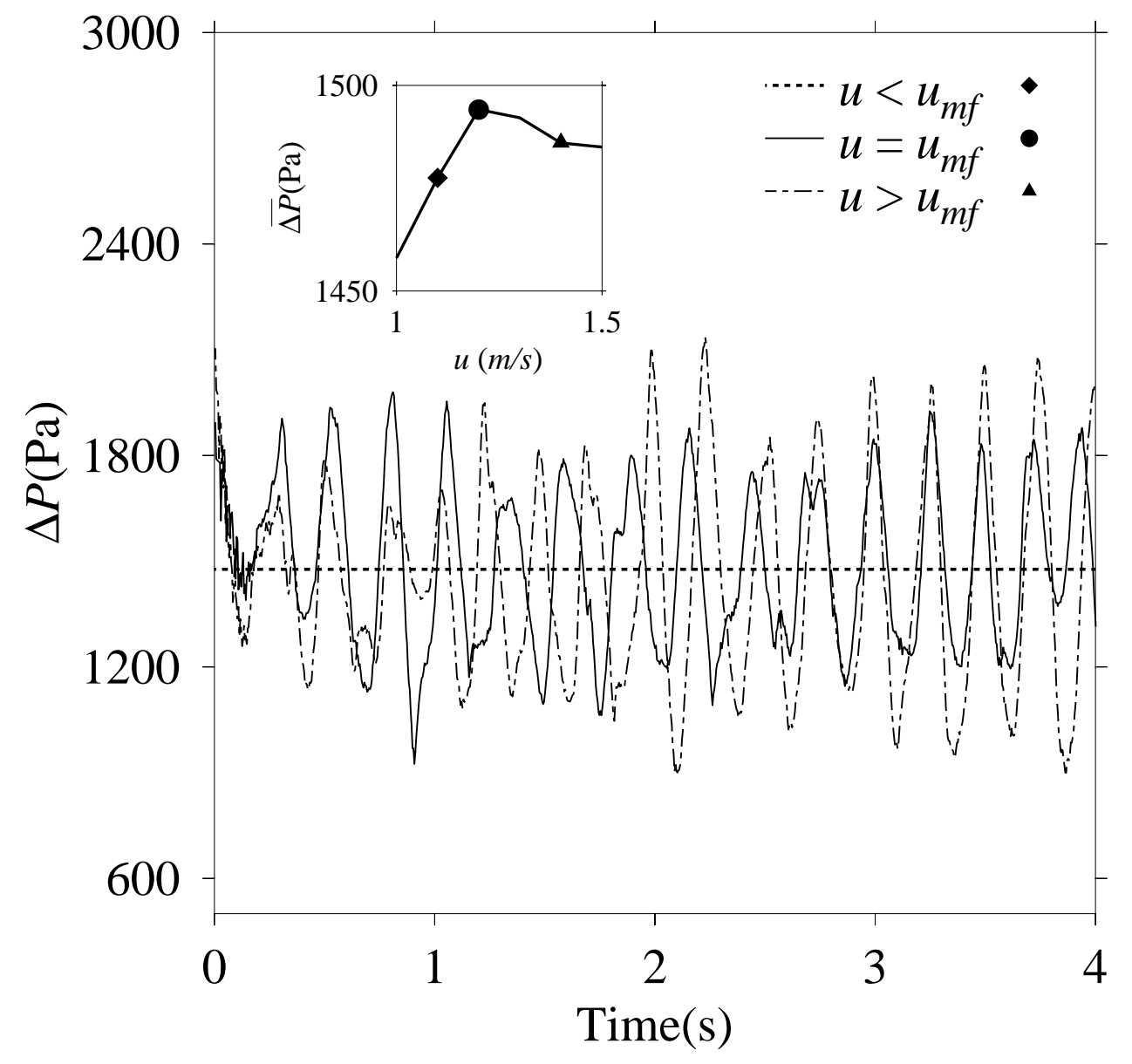

Figure 4.7 Pressure Drop versus Time for different gas velocities. Inset graph shows the variation of pressure drop with velocity. 


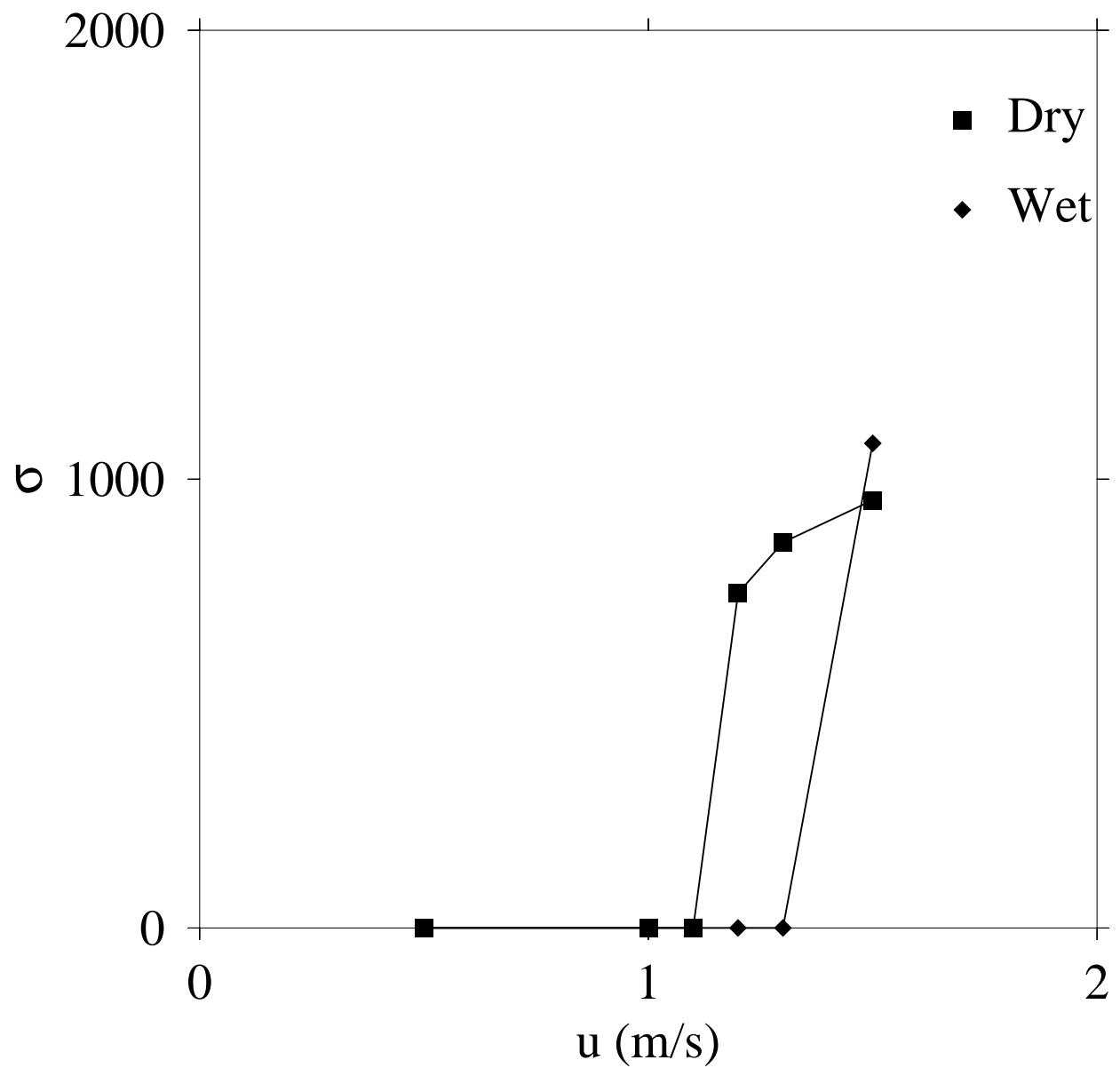

Figure 4.8 Standard Deviation of the Pressure Drop versus Gas-Velocity for dry and wet systems. 


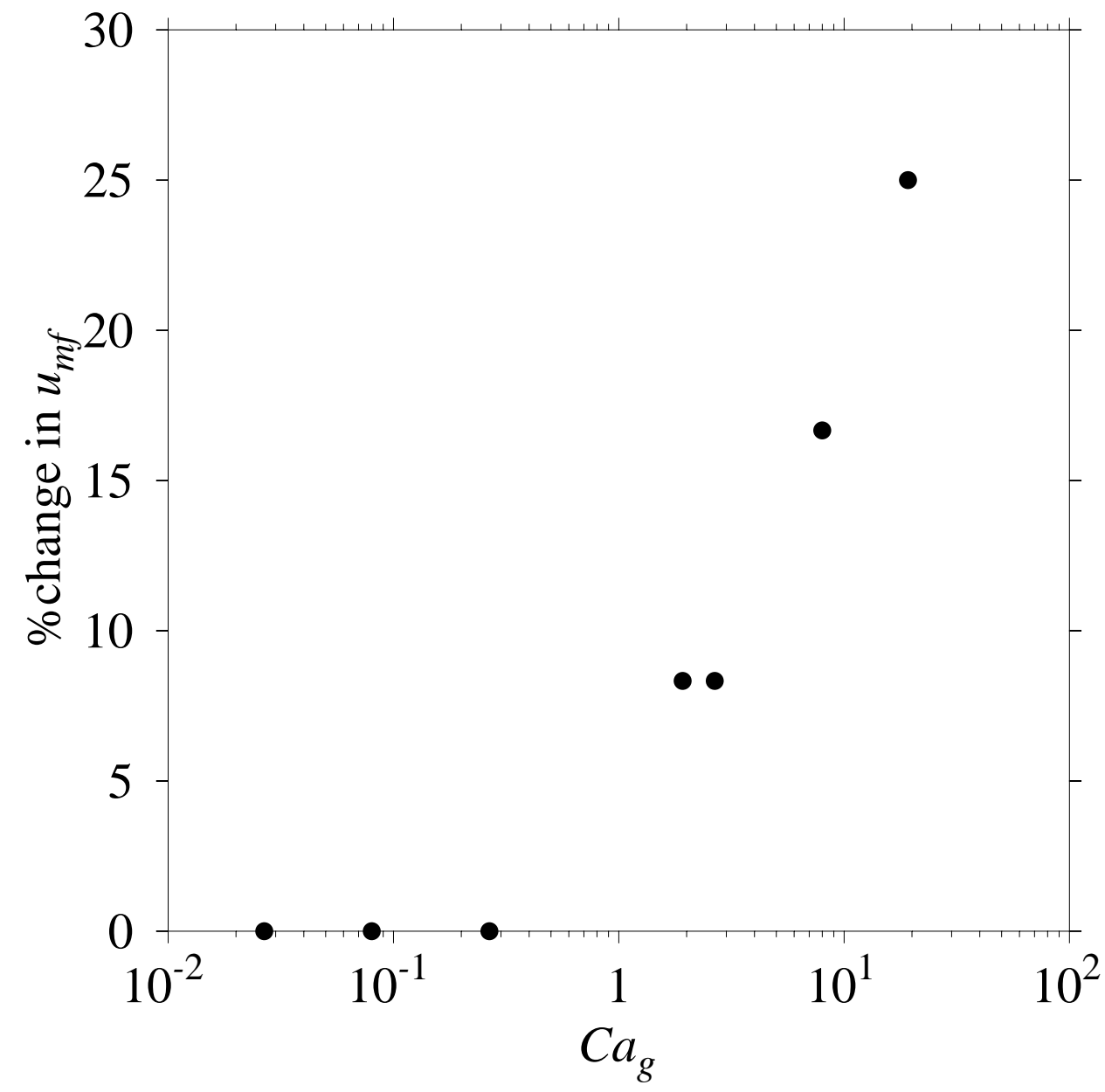

Figure 4.9 \%change in minimum fluidization velocity versus Granular Capillary Number 
fluidization velocity increase markedly requiring as much as a $30 \%$ increase in $u_{m f}$ at the highest $C a_{g}$ examined. 


\subsection{OUTLOOK}

Cohesive gas-solid flows are one of the least understood and at the same time, one of the most important areas in particle technology. Characterization tools are extremely useful for studying the practical applications of these flows as approximate behavior can be predicted based on the simple knowledge of equipment, gas and particle parameters with no need for costly eqxperimentation.

In this work we examined the transition from non-cohesive to cohesive behavior in gassolid systems. We show that, while $B o_{g}>1$ is a necessary condition for cohesive behavior, it is not a sufficient condition. In fact, a newly suggested dimensionless group, the Granular Capillary Number $\left(\mathrm{Ca}_{g}\right)$, must also be larger than unity. By looking at systems traditionally thought to be cohesive $\left(B o_{g}>1\right)$, we see a marked change in behavior as the $C a_{g}$ changes from non-cohesive $\left(C a_{g}<1\right)$ to cohesive $\left(C a_{g}>1\right)$ both in mixing rates and in fluidization behavior. This simple characterization tool may serve as a useful a priori test of the fluidization character to be expected in a wet gas-solid system; however, at present no allowance is made for particles with different properties, changes in liquid bridge volume, or wall effects.

As presented in this work, cohesion is induced in these beds through liquid bridges. The next step of this research should involve examining other inter particle forces, van der Waals and electrostatic forces. An extensive computational analysis of practical applications, in particular pneumatic conveying needs to be done; although proper choice of periodic boundary conditions in these simulations is unclear. Further, polydispersity in size, shape and properties should be explored as well. Finally, incorporation of TPD (Thermal Particle Dynamics) with a Discrete Particle simulation may allow the analysis of more industrially relevant problems where evaporation of moisture may take place. 


\section{BIBLIOGRAPHY}




\section{BIBLIOGRAPHY}

[1] C.Y. Wen and L.H. Chen. Flow modeling concepts of fluidized beds. In N.P. Cheremisinoff and R. Gupta, editors, The Handbook of Fluids in Motion, pages 665-714. 1983.

[2] M.J. Rhodes, X.S. Wang, M. Nguyen, P. Stewart, and K. Liffman. Onset of cohesive behavior in gas fluidized beds: a numerical study using dem simulation. Chem. Eng. Sci., 56:4433-4438, 2001.

[3] L.J. McLaughlin and M.J. Rhodes. Prediction of fluidized bed behavior in the presence of liquid bridges. Powder Technology, 114:213-223, 2001.

[4] S.T. Nase, W.L. Vargas, A.A. Abatan, and J.J. McCarthy. Discrete characterization tools for cohesive granular material. Powder Technology, 116:214-223, 2001.

[5] B. J. Ennis, J. Green, and R. Davies. The legacy of neglect. Chem. Eng. Prog., April:32-43, 1994.

[6] T. M. Knowlton, J.W. Carson, G.E. Klinzing, and W.C. Yang. The importance of storage, transfer, and collection. Chem. Eng. Prog., April:44-54, 1994.

[7] D. Geldart. Types of gas fluidization. Powder Technology, 7:285-292, 1973.

[8] J.L.R. Orband and D. Geldart. Direct measurement of powder cohesion using a torsional device. Powder Technology, 92:25-33, 1997.

[9] G. Lian, C. Thornton, and M.J. Adams. Discrete particle simulation of agglomerate impact coalescence. Chem. Eng. Sci., 53:3381-3391, 1998.

[10] T. Mikami, H. Kamiya, and M. Horio. Numerical simulation of cohesive powder in a fluidized bed. Chem. Eng. Sci., 53:1927-1940, 1998.

[11] J.F. Davidson. Symposium on fluidization-discussion. Trans. Inst. Chem. Engng., 39:1961, 223-240.

[12] T.B. Anderson and R. Jackson. A fluid mechanical description of fluidized beds. Ind. Eng. Chem. Fundam., 6:527-539, 1967.

[13] J.W. Pritchett, T.R. Blake, and S.K. Garg. A numerical model of gas fluidized beds. AIChE Symp. Ser. No. 176, 74:134-148, 1978.

[14] Y. Tsuji, T. Kawaguchi, and T. Tanaka. Discrete particle simulation of two-dimensional fluidized bed. Powder Technology, 77:79-87, 1993.

[15] J. Ding and D. Gidaspow. A bubbling fluidization model using kinetic theory of granular flow. AIChEJ, 36:523-528, 1990.

[16] M. Syamlal, W. Rogers, and T.J. O'Brien. MFIX documentation, Theory Guide, U.S. Dept. of Energy, Office of Fossil Energy, Tech. Note. 1993. 
[17] C.K.K. Lun, S.B. Savage, D.J. Jefferey, and N. Chepurniy. Kinetic theories for granular flow: inelastic particles in couette flow and slightly inelastic particles in a general flowfield. J. Fluid Mech., 140:223, 1984.

[18] P.A. Cundall and O.D.L. Strack. A discrete numerical model for granular assemblies. Géotechnique, 29:47-65, 1979.

[19] B.H. Xu and A.B. Yu. Numerical simulation of the gas-solid flow in a fluidized bed by combining discrete particle method with computational fluid dynamics. Chem. Eng. Sci., 52:2785-2809, 1997.

[20] C. Thornton and C. W. Randall. Applications of theoretical contact mechanics to solid particle system simulation. In M. Satake and J. T. Jenkins, editors, Micromechanics of Granular Material, pages 133-142. Elsevier Science Publishers, Amsterdam, 1988.

[21] J. Schäfer, S. Dippel, and E. Wolf. Force schemes in simulations of granular materials. J. Phys. I, 67:1751-1776, 1991.

[22] H. Hertz. Ueber die berhrungfester elastischer korper. J. renie ange. Math., 92:1-15, 1881.

[23] R.D. Mindlin. Compliance of elastic bodies in contact. J. Appl. Mech., 16:256-270, 1949.

[24] R.A. Fisher. On the capillary forces in an ideal soil. J. Agric. Sci., 16:491-505, 1926.

[25] B.J. Ennis, J. Li, G.I. Tardos, and R. Pfeffer. The influence of viscosity on the strength of an axially strained pendular liquid bridge. Chem. Eng. Sci., 45:3071-3088, 1990.

[26] M.J. Adams and V. Perchard. The cohesive forces between particles with interstitial fluid. Inst. Chem. Eng. Symp., 91:147-160, 1985.

[27] A.J. Goldman, R.G. Cox, and H. Brenner. Slow viscous motion of a sphere parallel to a plan wall i. motion through a quiescent fluid. Chem. Eng. Sci., 22:637-651, 1967.

[28] L. S. Fan and C. Zhu. Principles of Gas-Solid Flows. Cambridge University Press, New York, 1998.

[29] B.P.B. Hoomans, J.A.M. Kuipers, and W.P.M. van Swaaij. Granular dynamics simulation of segregation phenomena in bubbling gas-fluidized beds. Powder Technology, 109:41-48, 2000.

[30] J.A.M. Kuipers, K.J. van Duin, F.P.H. van Beckum, and W.P.M. van Swaaij. A numerical model for gas-fluidized beds. Chem. Eng. Sci., 47:1913, 1992.

[31] C.Y. Wen and H.Y. Yu. Mechanics of fluidization. Chem. Engng. Prog. Symp. Ser., 62:100, 1966.

[32] J.F. Richardson and W.N. Zaki. Sedimentation and fluidization: part 1. Trans. Inst. Chem. Engng., 32:35, 1954. 
[33] H.K. Versteeg and W. Malalasekera. An introduction to Computational Fluid Dynamics: The Finite Volume Method. John Wiley \& Sons Inc., New York, 1995.

[34] S.V. Patankar. Numerical Heat and Transfer and Fluid Flow. Hemisphere, New York, 1980.

[35] A. Munjiza and K.R.F. Andrews. NBS contact detection algorithm for bodies of similar size. Int. J. Numer. Meth. Engng., 43:131-149, 1998.

[36] B. J. Ennis, G. I. Tardos, and R. Pfeffer. A microlevel-based characterization of granulation phenomena. Powder Technol., 65:257-272, 1991.

[37] R.A. Bagnold. Experiments on a gravity-free dispersion of large solid spheres in a newtonian fluid under shear. Proc. R. Soc., 225:4-63, 1954.

[38] J. J. McCarthy, D. V. Khakhar, and J. M. Ottino. Computational studies of granular mixing. Pow. Technol., 109:72-82, 2000.

[39] M. Poux, P. Fayolle, J. Betrand, D. Bridoux, and J. Bousquet. Powder mixing: Some practical rules applied to agitated systems. Powder Technol., 68:213-234, 1991.

[40] K.D. Kafui, C. Thornton, and M.J. Adams. Discrete particle-continuum fluid modeling of gas-solid fluidized beds. Chem. Eng. Sci., 57:2395-2410, 2002. 